\title{
Magnetic Doppler imaging of the chemically peculiar star HD 125248*
}

\author{
N. Rusomarov ${ }^{1}$, O. Kochukhov ${ }^{1}$, T. Ryabchikova ${ }^{2}$, and I. Ilyin ${ }^{3}$ \\ ${ }^{1}$ Department of Physics and Astronomy, Uppsala University, Box 516, 75120 Uppsala, Sweden \\ e-mail: naum.rusomarov@gmail.com \\ 2 Institute of Astronomy, Russian Academy of Sciences, Pyatnistkaya 48, 119017 Moscow, Russia \\ 3 Astrophysikalisches Institut Potsdam, An der Sternwarte 16, 14482 Potsdam, Germany
}

Received 9 November 2015 / Accepted 25 January 2016

\begin{abstract}
Context. Intermediate-mass, chemically peculiar stars with strong magnetic fields provide an excellent opportunity to study the topology of their surface magnetic fields and the interplay between magnetic geometries and abundance inhomogeneities in the atmospheres of these stars.

Aims. We reconstruct detailed maps of the surface magnetic field and abundance distributions for the magnetic Ap star HD 125248. Methods. We performed the analysis based on phase-resolved, four Stokes parameter spectropolarimetric observations obtained with the HARPSpol instrument. These data were interpreted with the help of magnetic Doppler imaging techniques and model atmospheres taking the effects of strong magnetic fields and nonsolar chemical composition into account.

Results. We improved the atmospheric parameters of the star, $T_{\text {eff }}=9850 \pm 250 \mathrm{~K}$ and $\log g=4.05 \pm 0.10$. We performed detailed abundance analysis, which confirmed that HD 125248 has abundances typical of other Ap stars, and discovered significant vertical stratification effects for the Fe II and CrII ions. We computed LSD Stokes profiles using several line masks corresponding to Fepeak and rare earth elements, and studied their behavior with rotational phase. Combining previous longitudinal field measurements with our own observations, we improved the rotational period of the star $P_{\text {rot }}=9.29558 \pm 0.00006 \mathrm{~d}$. Magnetic Doppler imaging of HD 125248 showed that its magnetic field is mostly poloidal and quasi-dipolar with two large spots of different polarity and field strength. The chemical maps of $\mathrm{Fe}, \mathrm{Cr}, \mathrm{Ce}, \mathrm{Nd}, \mathrm{Gd}$, and $\mathrm{Ti}$ show abundance contrasts of 0.9-3.5 dex. Among these elements, the Fe abundance map does not show high-contrast features. $\mathrm{Cr}$ is overabundant around the negative magnetic pole and has 3.5 dex abundance range. The rare earth elements and $\mathrm{Ti}$ are overabundant near the positive magnetic pole.

Conclusions. The magnetic field of HD 125248 has strong deviations from the classical oblique dipole field geometry. A comparison of the magnetic field topology of HD 125248 with the results derived for other stars using four Stokes magnetic Doppler imaging suggests evidence that the field topology becomes simpler with increasing age. The abundance maps show weak correlation with magnetic field geometry, but they do not agree with the theoretical atomic diffusion calculations, which predict element accumulation in the horizontal field regions.
\end{abstract}

Key words. stars: abundances - stars: atmospheres - stars: magnetic field - stars: chemically peculiar - stars: individual: HD 125248

\section{Introduction}

The star HD 125248 (CS Vir, HR 5355) is one of the most outstanding objects in the class of magnetic chemically peculiar stars. This object was discovered by Morgan (1931) to be a strong spectrum variable of type Alp $\mathrm{SrCrEu}$ (Renson \& Manfroid 2009) with a period of $\simeq 9.3$ days (Deutsch 1947). Morgan (1931) observed that the absorption lines of CrII and Eu II varied in antiphase to each other, while the lines of $\mathrm{Fe}$ and $\mathrm{Ti}$ hardly showed any variation. Observations by Babcock (1947) showed that HD 125248 possesses a strong magnetic field, whose line-of-sight component varies with the rotational period of the star between approximately -2 and $+2 \mathrm{kG}$ (Babcock 1951). Since then a considerable number of magnetic field measurements have been obtained for HD 125248, spanning more than half a century; the latest study is by Shorlin et al. (2002, see Sect. 4.1 for references). An equally substantial number of photometric studies exist (see

\footnotetext{
* Based on observations collected at the European Southern Observatory, Chile (ESO programs 088.D-0066, 090.D-0256).
}

Mikulášek et al. 2007 for references), showing that HD 125248 is variable with the same period in the visible and near-infrared light, spectrum, and magnetic field (e.g., Catalano et al. 1992, 1998; Leone \& Catanzaro 2001). Recent spectroscopic studies by Mathys (1992) and Gonzalez \& Artru (1994) have also investigated the variation of oxygen lines with the rotational period of the star.

HD 125248 was the first star for which Stibbs (1950) proposed the oblique rotator model (ORM) as a way to explain the observed variations of photometric, spectral, and magnetic field observables. In this framework, the magnetic field is frozen into the atmosphere of the star, with its axis not aligned with the rotational axis of the star, resulting in a time-dependent magnetic configuration as seen by the observer. HD 125248 is also the first object for which an attempt at spherical harmonics analysis was performed with the aim of producing surface maps of the abundance anomalies and magnetic field (Deutsch 1957). The latest study of the magnetic field of HD 125248 was produced by Bagnulo et al. $(1999,2002)$ with low-resolution circular polarization spectra (Stokes $I$ and $V$ ). The study found that the 
magnetic field of HD 125248 shows strong deviations from an oblique dipole field. However, Bagnulo et al. (1999) could not find a unique solution for the more complex model represented by a superposition of a dipole and a quadrupole.

It is evident that HD 125248 is an excellent candidate for magnetic Doppler imaging (MDI; Piskunov \& Kochukhov 2002; Kochukhov \& Piskunov 2002). This method can simultaneously reconstruct the surface abundance distribution of different chemical elements and restore the vector magnetic field at the stellar surface. Magnetic Doppler imaging based on the full Stokes vector spectropolarimetric observations is particularly powerful as it does not require a priori information about the (global) geometry of a magnetic field in contrast to MDI based only on circular polarization data. Recent four Stokes parameter MDI studies of magnetic Ap stars have revealed field topologies that depart significantly from oblique dipole models and even contain some small-scale magnetic structures $\left(\alpha^{2} \mathrm{CVn}\right.$; Kochukhov \& Wade 2010; Silvester et al. 2014a; HD 32633, Silvester et al. 2015; 53 Cam, Kochukhov et al. 2004).

We obtained high-resolution spectropolarimetric observations of HD 125248 as part of our program aimed at observing Ap stars in all four Stokes parameters with the HARPSpol instrument (Piskunov et al. 2011) at the ESO $3.6 \mathrm{~m}$ telescope. The project provides insight into the mechanism of atomic diffusion in the presence of magnetic fields (Michaud et al. 1981; Alecian \& Stift 2010), which is believed to be responsible for the appearance of horizontal (spots) and vertical (stratification) chemical abundance inhomogeneities. Moreover, Braithwaite \& Nordlund (2006) have shown that stable magnetic fields can exist in the interiors of main-sequence stars with radiative envelopes. In this context, detailed empirical information on the geometry of magnetic fields of $\mathrm{Ap} / \mathrm{Bp}$ stars gathered in a systematic approach is essential for understanding the underlying magnetohydrodynamic processes that lead to the generation and evolution of global magnetic fields in the interiors of mainsequence stars with radiative envelopes.

The paper is organized as follows. Section 2 describes the observations. In Sect. 3 we derive the fundamental parameters of the star. Section 4 discusses the polarization signatures in the mean lines profiles, the measurements of the integral magnetic observables, and the rotational period search. Section 5 introduces the principles of MDI, describes the choice of spectral lines used in the analysis, and the determination of rotational velocity and orientation of the rotational axis. The resulting magnetic and chemical maps are discussed in Sect. 6. The summary and discussion are presented in Sect. 7.

\section{Observations}

Spectropolarimetric observations of HD 125248 were obtained at the ESO $3.6 \mathrm{~m}$ telescope with the HARPS spectrograph (Mayor et al. 2003) and its polarimetric unit HARPSpol (Piskunov et al. 2011; Snik et al. 2011). We acquired 36 individual Stokes parameter observations spread over 12 observing nights for two consecutive years starting from 2012. These observing runs yielded a good phase coverage over the entire rotational period of the star.

A spectropolarimetric observation of an individual Stokes parameter consists of four subexposures obtained with different orientations of the quarter-wave (Stokes $V$ ) or half-wave (Stokes $Q$ and $U$ ) retarder plate. From one such sequence of observations, we derive an intensity spectrum (Stokes $I$ ) and one more Stokes $Q, U$, or $V$ parameter. The eight extracted one-dimensional spectra corresponding to four subexposures were combined using the ratio method (Donati et al. 1997; Bagnulo et al. 2009). This method has the advantage of automatically producing a null spectrum that can be used for assessing spurious polarization and crosstalks.

The spectra have resolving power, $\lambda / \Delta \lambda$, of approximately 110000 and a coverage of $3780-6910 \AA$ with an $80 \AA$ gap located at $5290 \AA$. The exposure time of each subexposure during the first observing run in 2012 was set to $500 \mathrm{~s}$. In order to compensate for the worse seeing experienced during the second observing run in 2013 each subexposure had an exposure time between 600 and 900 seconds. The signal-to-noise ratio $(\mathrm{S} / \mathrm{N})$ measured for all observations at $5500 \AA$ is $150-350$. We note that for two nights (22 and $23 \mathrm{Feb}$. 2013) the $\mathrm{S} / \mathrm{N}$ for the blue part of the spectrum is less than 100 , which was caused by highly variable seeing. The journal of observations is provided in Table 1.

The observational and data processing techniques applied to the spectra for HD 125248 are identical to those described by Rusomarov et al. (2013). Those authors provide a detailed discussion of the observation procedures and reduction steps, such as extraction of the spectra, calculation of the Stokes parameters, normalization of the resulting spectra, and a more complete description of the instrument.

In addition to the HARPSpol four Stokes parameter spectra we have analyzed nine circular polarization observations of HD 125248 collected in June 2001 with the help of the SOFIN spectropolarimeter at the Nordic Optical Telescope. These data have a resolving power of $\lambda / \Delta \lambda=70000$ and provide an incomplete wavelength coverage of the 4770-7090 A interval. The typical peak S/N of these spectra is $200-300$ for $\lambda>5000 \AA$. The reduction of the SOFIN spectra closely followed the procedure described by Lüftinger et al. (2010). Since the HARPSpol spectra already provided a good phase coverage with superior spectral resolution, the SOFIN data was used solely for determination of the mean longitudinal magnetic field.

\section{Fundamental parameters of HD 125248}

The most recent effective temperature estimate for HD 125248 , $T_{\text {eff }}=9500 \mathrm{~K}$, was obtained by Lipski \& Stępień (2008) from a fit of metal-enhanced model atmospheres to the observed spectral energy distributions (SED) in the ultraviolet (UV) and optical regions. However, the authors assumed a surface gravity $\log g=4.0$ and an increased metallicity by 0.5 dex relative to the Sun, which raises some concern about the accuracy of the study because the phase averaged mean spectrum shows metal lines that are significantly stronger than what was assumed by Lipski \& Stępień (2008). In an earlier study, Monier (1992) found that the energy distribution of HD 125248 is best reproduced by an atmosphere model with a slightly higher temperature, $T_{\text {eff }}=9700 \mathrm{~K}$, and a surface gravity $\log g=4.25$ with $[\mathrm{M} / \mathrm{H}]=+1.0 \mathrm{dex}$.

We started the atmospheric analysis by computing a model atmosphere with the same values of $T_{\text {eff }}, \log g$, and chemical composition as used by Lipski \& Stępień (2008), with the LLMODELS code (Shulyak et al. 2004), which incorporates individual elemental abundances and has detailed treatment of line opacities due to Zeeman splitting and polarized radiative transfer (Khan \& Shulyak 2006). The strong magnetic field of HD 125248 is expected to suppress all convective motions in the atmosphere, therefore we set the micro- and macroturbulent velocities to zero in all calculations.

We adopted a radial magnetic field with $7.2 \mathrm{kG}$ strength by phase averaging the mean field modulus of HD 125248, 
Table 1. Journal of spectropolarimetric observations of HD 125248.

\begin{tabular}{lcccccccccc}
\hline \hline \multirow{2}{*}{ UT Date } & \multicolumn{3}{c}{ HJD (2455000+) } & \multirow{2}{*}{\begin{tabular}{c}
\multirow{2}{*}{$S$} \\
\end{tabular}} & $Q$ & $U$ & \multicolumn{3}{c}{$S / N$} & \multicolumn{3}{c}{ Median $S / N$} \\
& 28 Mar. 2012 & 1014.7988 & 1014.8235 & 1014.7735 & 0.704 & 0.003 & $136-442$ & 317 & 309 & 326 \\
29 Mar. 2012 & 1015.7721 & 1015.7968 & 1015.7465 & 0.808 & 0.003 & $101-402$ & 277 & 304 & 250 \\
30 Mar. 2012 & 1016.8004 & 1016.8251 & 1016.7749 & 0.919 & 0.003 & $122-411$ & 297 & 309 & 292 \\
31 Mar. 2012 & 1017.7727 & 1017.7975 & 1017.7475 & 0.023 & 0.003 & $105-389$ & 264 & 294 & 257 \\
01 Apr. 2012 & 1018.8119 & 1018.8555 & 1018.7772 & 0.136 & 0.006 & $100-479$ & 331 & 360 & 228 \\
02 Apr. 2012 & 1019.7723 & 1019.8109 & 1019.7369 & 0.239 & 0.005 & $116-479$ & 358 & 297 & 278 \\
22 Feb. 2013 & 1345.8471 & 1345.8774 & 1345.8161 & 0.317 & 0.004 & $91-338$ & 234 & 252 & 225 \\
23 Feb. 2013 & 1346.8530 & 1346.8893 & 1346.8193 & 0.425 & 0.005 & $63-314$ & 155 & 209 & 228 \\
24 Feb. 2013 & 1347.8547 & 1347.8933 & 1347.8174 & 0.533 & 0.005 & $104-361$ & 258 & 268 & 258 \\
25 Feb. 2013 & 1348.8597 & 1348.9030 & 1348.8179 & 0.641 & 0.006 & $120-449$ & 307 & 331 & 295 \\
26 Feb. 2013 & 1349.8598 & 1349.9007 & 1349.8213 & 0.749 & 0.005 & $116-478$ & 344 & 356 & 288 \\
27 Feb. 2013 & 1350.8502 & 1350.8935 & 1350.8105 & 0.855 & 0.006 & $111-398$ & 290 & 296 & 272 \\
\hline
\end{tabular}

Notes. First column gives the UT date at the beginning of each observing night. Heliocentric Julian Dates (HJD) at mid-exposure for each observed Stokes parameter are given in Cols. 2-4. Mean phase, $\bar{\varphi}$, and the maximum difference, $\delta \varphi$, between $\bar{\varphi}$ and phases of individual Stokes parameter observations are presented in Cols. 5, 6. Rotational phases were calculated according to our improved ephemeris (Sect. 4.1). The range of the S/N for each group of Stokes parameter observations taken during one night is given in Col. 7. The median S/N for each individual Stokes parameter observation are presented in Cols. 8-10. The median $\mathrm{S} / \mathrm{N}$ was calculated using several echelle orders around $\lambda=5500 \AA$, where maximum counts were reached.

calculated from the dipole plus quadrupole models obtained by Bagnulo et al. (2002). The adopted estimate is close to the $6.6 \mathrm{kG}$ value predicted by the model of Glagolevskij (2007). The Vienna atomic line database (VALD) was used as a source of atomic data (Kupka et al. 1999). We used this model atmosphere and estimated the abundances of a number of light elements and Fe-peak and rare earth elements. For this purpose we fitted synthetic profiles calculated with the SYNMAST code (Kochukhov et al. 2010) to the phase-averaged intensity (Stokes I) spectrum of HD 125248.

The abundances determined in this step were then used to compute a grid of model atmospheres and SEDs with $T_{\text {eff }}=9000-10200 \mathrm{~K}$ and $\log g=3.9-4.3$ in $200 \mathrm{~K}$ steps for the effective temperature and 0.05 dex steps for the surface gravity. The calculated flux distributions were then compared to spectrophotometric observations in the optical range (Adelman et al. 1989), IUE Newly Extracted Spectra (IUE NES) in the UV obtained with large aperture, and near-IR photometric observations (2MASS fluxes). We adopted zero interstellar reddening for HD 125248, following Lipski \& Stępień (2008) who assumed that stars closer than $100 \mathrm{pc}$ do not experience significant interstellar absorption. We note, however, that their value of $d=90 \mathrm{pc}$ results from a parallax of $\pi=11.08 \pm 0.91$ mas from an earlier version of the Hipparcos catalog (Perryman et al. 1997). The latest estimate for the parallax of HD 125248 is $\pi=9.80 \pm 0.67$ mas (van Leeuwen 2007), suggesting a distance of $102 \mathrm{pc}$, which is still close to $100 \mathrm{pc}$.

The SED fitting procedure is illustrated in Fig. 1. We found that an effective temperature of $T_{\text {eff }}=9850 \pm 250 \mathrm{~K}$ most accurately describes the slope of the Paschen continuum, the Balmer jump, and the UV SED of HD 125248. This value is fairly insensitive to the choice of surface gravity and agrees well with previous studies. Changing the effective temperature by $250 \mathrm{~K}$ in each direction improves the fit in the UV or near-IR region slightly, therefore we consider this error bar to be a good estimate of the uncertainties of our fitting procedure.

Given the effective temperature determined in the previous step we constrained the surface gravity $\log g=4.05 \pm 0.10$ by fitting synthetic profiles computed with SYNMAST to the observed Balmer lines. The comparison between the observed and calculated profiles of $\mathrm{H} \alpha, \mathrm{H} \beta$, and $\mathrm{H} \gamma$ is presented in Fig. 2. The figure illustrates that the revised value of surface gravity describes the wings of the Balmer lines with good accuracy.

We combined the parallax of the star, $\pi=9.80 \pm 0.67$ mas, (van Leeuwen 2007) with its angular diameter, which was obtained in the SED fitting procedure, and derived the stellar radius $R=2.23 \pm 0.19 R_{\odot}$. This value agrees well with the previous estimates by Lipski \& Stępień $\left(2008, R=2.1 R_{\odot}\right)$ and Bagnulo et al. $\left(2002, R=1.97 R_{\odot}\right)$. The luminosity of HD 125248 was estimated using the standard relation between the stellar radius and effective temperature and was found to be $L=42.0 \pm 8.3 L_{\odot}$, which is within one sigma in comparison to the value determined by Kochukhov \& Bagnulo (2006, $\left.L=31.6 \pm 5.8 L_{\odot}\right)$. The new values of the surface gravity and radius imply a mass $M=2.0 \pm 0.6 M_{\odot}$, which is in good agreement with the mass estimate from stellar evolutionary models by Kochukhov \& Bagnulo $\left(2006, M=2.27 \pm 0.07 M_{\odot}\right)$. Given the good agreement of the fundamental parameters between this study and the paper by Kochukhov \& Bagnulo (2006), we deemed a redetermination of the age of HD 125248 from evolutionary tracks to be unnecessary.

Finally, the projected rotational velocity was estimated to be $v_{\mathrm{e}} \sin i=11.5 \pm 1.5 \mathrm{~km} \mathrm{~s}^{-1}$ by fitting the Stokes $I$ profiles of $\mathrm{Fe} \mathrm{I} / \mathrm{II}$ lines with low magnetic sensitivity. This value of $v_{\mathrm{e}} \sin i$ agrees well with the determinations by Abt \& Morrell (1995, $\left.v_{\mathrm{e}} \sin i=10 \mathrm{~km} \mathrm{~s}^{-1}\right)$ and Mathys $\left(1995, v_{\mathrm{e}} \sin i=9.7 \mathrm{~km} \mathrm{~s}^{-1}\right)$.

The fundamental parameters of HD 125248 are summarized in Table 2.

\subsection{Abundance analysis}

For the determination of accurate fundamental parameters of HD 125248 it was necessary to determine the abundance of many chemical elements. For this purpose we estimated the abundance of most light elements, iron-peak and rare earth elements, and several s-process elements. The mean abundances are summarized in Table 3. In short, the abundances of HD 125248 appear to follow the general trend for other Ap stars. Several light 
UV and optical

IR

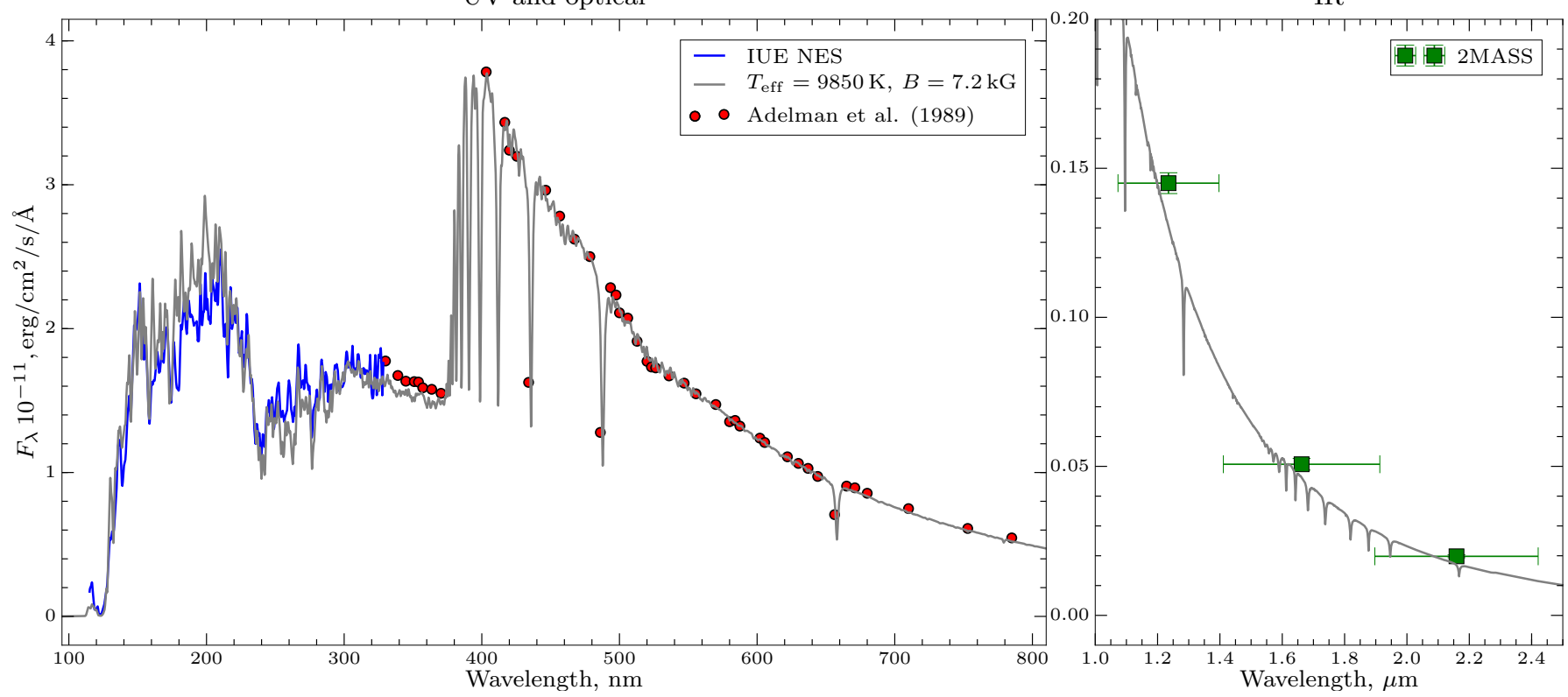

Fig. 1. Comparison between the theoretical and observed spectrophotometry of HD 125248. The light gray curve shows the spectral energy distribution computed using a model atmosphere with $T_{\text {eff }}=9850 \mathrm{~K}, \log g=4.05$, including the effects of individual nonsolar abundances of HD 125248 and a magnetic field with strength $\langle B\rangle=7200 \mathrm{G}$. The observations cover the UV (blue lines), optical (red circles) and near-IR (green squares) spectral regions.
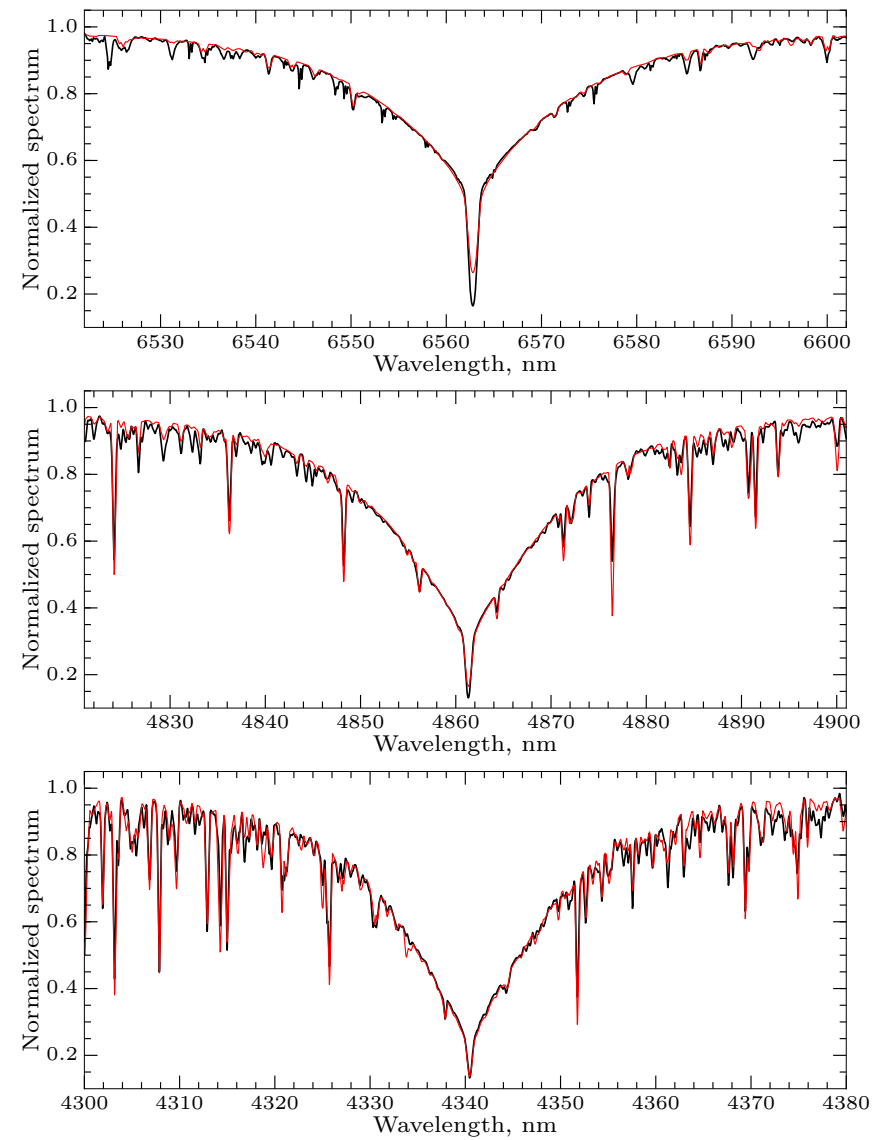

Fig. 2. Comparison between the observed (thick black curve) and computed (thin red curve) profiles of the $\mathrm{H} \alpha, \mathrm{H} \beta$, and $\mathrm{H} \gamma$ lines in the spectrum of HD 125248.

elements are slightly deficient, the Fe-peak elements are overabundant by about one dex with the exception of $\mathrm{Cr}$, which is
Table 2. Fundamental parameters of HD 125248.

\begin{tabular}{lll}
\hline \hline Parameter & Value & Reference \\
\hline$T_{\text {eff }}(\mathrm{K})$ & $9850 \pm 250$ & This study \\
$\log g$ & $4.05 \pm 0.10$ & This study \\
$L / L_{\odot}$ & $42.0 \pm 8.3$ & This study \\
$R / R_{\odot}$ & $2.23 \pm 0.19$ & This study \\
$M / M_{\odot}$ & $2.0 \pm 0.6$ & This study \\
$\log (t)(\mathrm{yr})$ & $8.37(7.94-8.53)$ & Kochukhov \& Bagnulo (2006) \\
\hline
\end{tabular}

overabundant by about two dex, while the rare earth elements are overabundant on average between two and three dex relative to their solar values.

We report abundances in $\log \left(N_{\mathrm{X}} / N_{\text {tot }}\right)$ units to be consistent with similar abundance and MDI studies of Ap stars. We note that in our analysis we did not account for hyperfine splitting or non-local thermodynamic equilibrium (non-LTE) effects, which can lead to systematic errors for certain elements.

\subsubsection{Light elements: $\mathrm{He}$ to $\mathrm{Ca}$}

The He abundance was obtained from two groups of He I lines at $\lambda 4471 \AA$ and $\lambda 5875 \AA$. A calculation assuming solar helium abundance produces He lines that appear to be too deep contrary to what is observed in the mean spectrum of HD 125248. Therefore, we adopted abundance $\log \left(\mathrm{He} / N_{\text {tot }}\right)=-1.80$, which is smaller by around five times compared to the solar value. For CNO elements we could only measure the abundance of $\mathrm{O}$, which appears to be slightly deficient by 0.3 dex. Abundances of other light elements are mostly solar, with the exception of $\mathrm{Mg}$ and $\mathrm{Si}$. The former is strongly depleted by about $0.8 \mathrm{dex}$, while the latter is slightly overabundant by 0.3 dex. 
Table 3. Mean atmospheric abundances of the Ap star HD 125248.

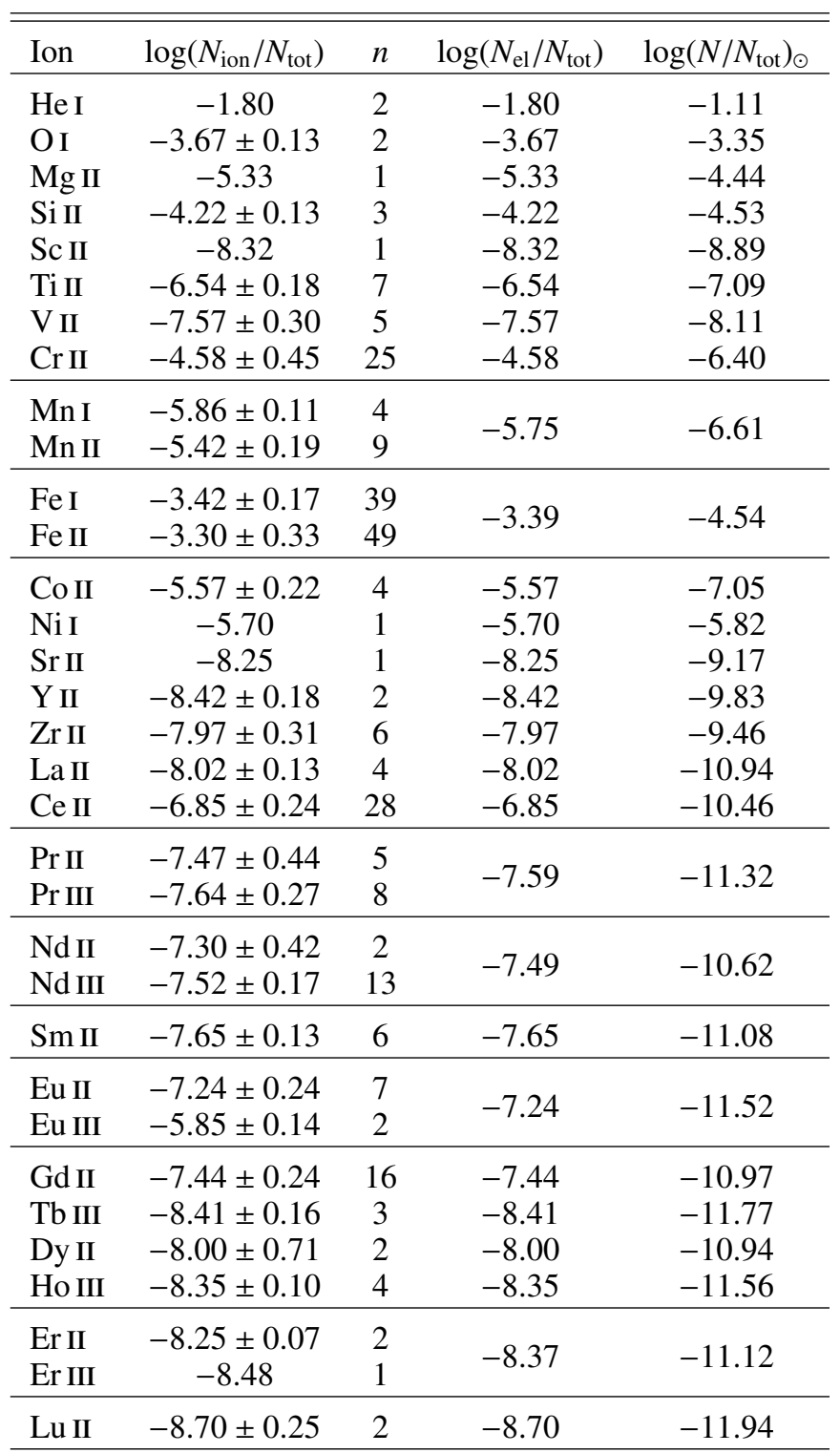

Notes. The first column identifies the ions for which we estimated the abundance given in the second column. The number of lines analyzed for each species is given in the third column. The adopted abundances for the corresponding chemical elements are given in column four. The solar abundances reported by Asplund et al. (2009) are given in the last column for comparison.

\subsubsection{Iron peak elements: $\mathrm{Sc}$ to $\mathrm{Ni}$}

The abundance of Sc was inferred from the Sc I $4415 \AA$ line, which requires $\log \left(\mathrm{Sc} / N_{\text {tot }}\right)=-8.32$ to reproduce the line profile. The Ti abundance was straightforward to infer, thanks to the seven Ti II lines that we could successfully reproduce with $\log \left(\mathrm{Ti} / N_{\text {tot }}\right)=-6.54$. Vanadium, in contrast to Ti, had a few blended V II lines, which yielded an abundance higher by $0.5 \mathrm{dex}$ relative to the solar value. However, the large error makes this result somewhat inconclusive. We derived the $\mathrm{Mn}$ abundance from MnI and Mn II lines. Both estimates indicate that Mn is overabundant, with the abundance for Mn II being $0.44 \mathrm{dex}$ higher than that for MnI. It is possible that this discrepancy is caused by hyperfine splitting. Cobalt has an overabundance of 1.55 dex, which might be caused by lack of accurate oscillator

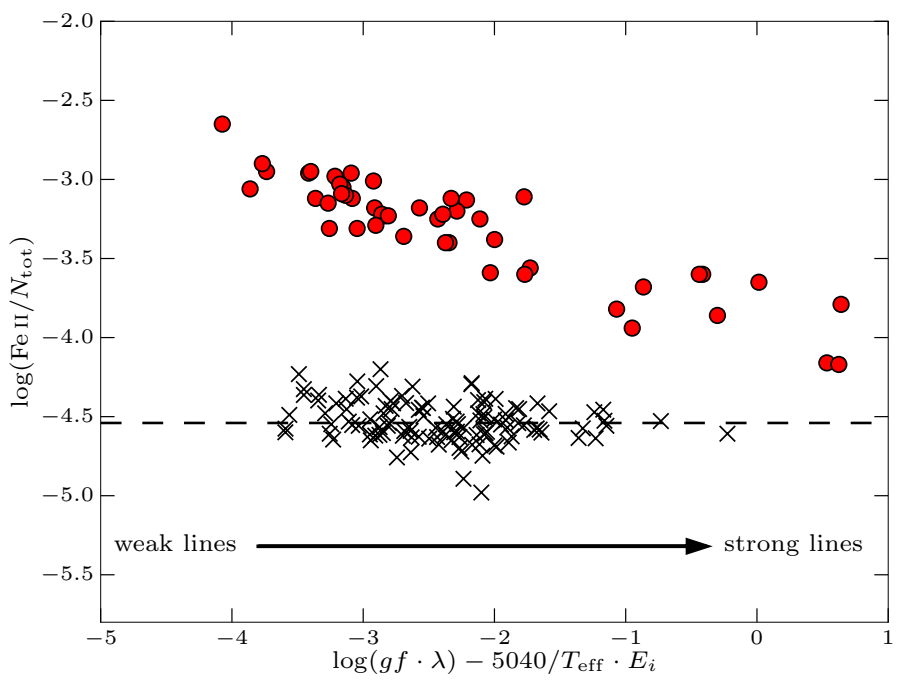

Fig. 3. Individual Fe II abundances as a function of line intensity in the atmospheres of HD 125248 and $21 \mathrm{Peg}$. The filled circles represent Fe II abundances for HD 125248. The data for 21 Peg are plotted using "x" symbols. The solar Fe abundance is shown with a dashed line.

strengths for Co II lines, or possibly, ignoring the hyperfine structure. Nickel, on the other hand, had only one Ni I $\lambda 4980.166 \AA$ line that was not distorted by blends. An attempt to measure the $\mathrm{Ni}$ abundance from this line gave an estimate that is only $0.1 \mathrm{dex}$ higher than the solar value. The attempt to measure the $\mathrm{Cr}$ abundance resulted in $\log \left(\mathrm{Cr} / N_{\text {tot }}\right)=-4.58$, which is by $\sim 2$ dex higher than in the Sun.

Considering the importance of $\mathrm{Fe}$ in stellar atmospheres calculations, we took special care to measure its abundance from a large number of $\mathrm{Fe} \mathrm{I}$ and $\mathrm{Fe}$ II lines with excitation energy in the range $1<E_{i}<12 \mathrm{eV}$. The measurements from Fe I and Fe II appear to agree well within the error bars with only a 0.12 dex difference between them. The larger scatter of the Fe II-based abundance led us to explore further the relationship between the abundance measured from individual lines versus the line strength expressed as a function of the oscillator strength and excitation energy (see, e.g., Ryabchikova 2014). We illustrate this in Fig. 3 for the Fe II lines. For comparison we added data for the normal A-type star 21 Peg (Table 9 of Fossati et al. 2009), which has an effective temperature similar to HD 125248. As we can see from the figure, there is a visible trend of abundance with the line strength; strong lines that are formed higher in the atmosphere give smaller individual abundances compared to the weak lines, which are formed near the photosphere. The presence of such a slope in abundance analysis of normal stars indicates that the effective temperature has been determined incorrectly. In the case of HD 125248, it is impossible to remove this trend by correcting the effective temperature without contradicting the spectrophotometry of this star. The same technique was applied to the individual abundances of $\mathrm{Cr}$ II lines, which have a very significant 0.45 dex scatter around the mean value, in spite of our best efforts to select mostly unblended $\mathrm{Cr}$ lines with various excitation energy and $\log g f$ factors. The analysis yielded similar results; the abundances from individual Cr II lines show a strong dependence on the line strength. We consider these results to be a clear sign of vertical stratification of chemical elements. Detailed investigation of vertical abundance stratification is beyond the scope of our paper. 


\subsubsection{Neutron capture elements: $\mathrm{Sr}, \mathrm{Y}$, and $\mathrm{Zr}$}

The Sr abundance was determined from the Sr II $\lambda 4215 \AA$ line to be enhanced by 0.9 dex compared to the solar value. We measured the Y abundance from two mostly unblended Y II lines, which require almost 1.5 dex overabundance to properly reproduce the observed profiles in the mean spectrum. The zirconium abundance was reliably deduced from six $\mathrm{Zr}$ II lines to be also overabundant by 1.5 dex. Our attempts at abundance analysis of other elements from the fifth group of the periodic table of elements were fruitless as we could not find spectral lines suitable for accurate abundance determination.

\subsubsection{Rare earth elements: $\mathrm{La}$ to $\mathrm{Lu}$}

The rare earth elements appear to be significantly enhanced by $\sim 3$ dex and more compared to the solar values.

For some rare earth elements it was possible to estimate their abundance using lines of the first and second ionization stages. These estimates demonstrated a very good agreement between abundances derived from the lines of different ions of the same element. The only exception to this rule is Eu, which shows significantly different values when determined from Eu II and Eu III lines. The Eu abundance derived from Eu II lines is similar to other rare earth elements, while the Eu III abundance is higher by 1.4 dex. The Eu III abundance, unfortunately, is less constrained because we could only determine it from the Eu III $\lambda 5376 \AA$ and Eu III $\lambda 6666 \AA$ lines. A similar pattern with the Eu anomaly and the absence of other rare earth anomalies has been observed in the atmosphere of another Ap star HD 144897 (Ryabchikova et al. 2006) although more lines of Eu III were used in abundance analysis of this star. The lack of reliable atomic data (hfs constants, isotopic shifts, collision rates) for Eu III does not allow us to conclude whether this discrepancy is caused by ignoring the hyperfine splitting and non-LTE effects (Mashonkina et al. 2002), or by an actual physical mechanism that produces abundance differences between ionization states of this particular element, atomic diffusion, for example.

\section{Integral magnetic observables}

The mean characteristics of a stellar magnetic field, such as the mean longitudinal field, can be inferred directly from spectropolarimetric observations (e.g., Landstreet \& Mathys 2000; Bagnulo et al. 2002). Such measurements of the magnetic observables for HD 125248 from polarization signals in individual lines are difficult because its spectrum is highly complex; most spectral line profiles are severely blended by an amount that changes significantly with rotational phase. Therefore, we employed the least-squares deconvolution technique (LSD; Donati et al. 1997) to the Stokes spectra of HD 125248. This technique assumes that a stellar spectrum can be represented as a superposition of similar profiles scaled by a factor that depends on the line strength, the magnetic sensitivity, and the central wavelength. For this study, we used the multiprofile version of the LSD method introduced by Kochukhov et al. (2010), which allows us to disentangle the mean profiles of a given set of elements while minimizing blending of the lines of other elements.

We used the VALD3 database (Ryabchikova et al. 2015) as a source of atomic data necessary for construction of the LSD line mask, together with the model atmosphere and abundance table produced in Sect. 3. After removing spectral lines affected by hydrogen and telluric lines, and lines with central depth less

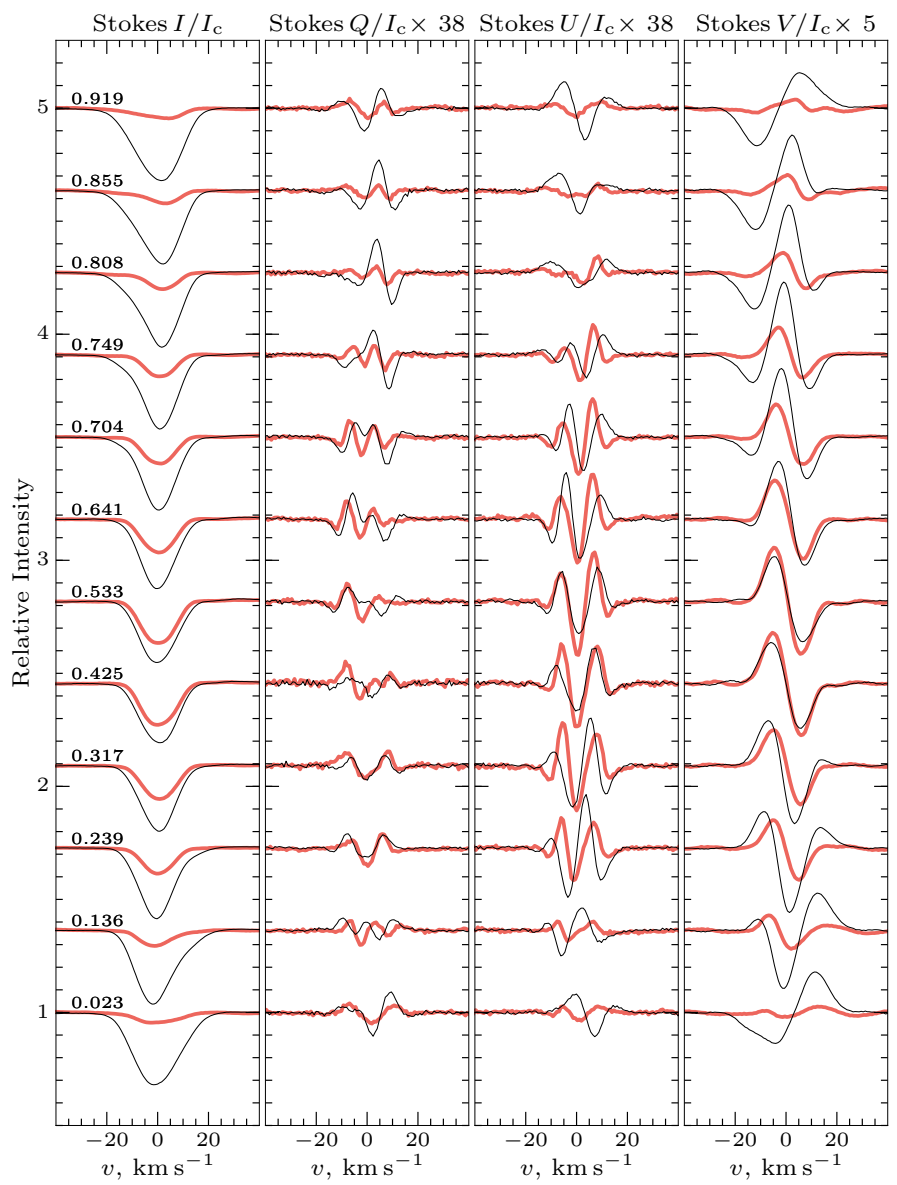

Fig. 4. Stokes $I$ (first panel), $Q$ (second panel), $U$ (third panel), and $V$ (fourth panel) LSD profiles of HD 125248. The spectra are shifted vertically according to the rotational phases indicated in the first panel. The Stokes $Q U$ profiles have been rescaled by a factor of 38 , and the Stokes $V$ profiles by a factor of 5 to match the amplitude of the Stokes $I$ profiles. The LSD profiles of Fe-peak elements are illustrated with thin lines (black), while the LSD profiles of the rare earth elements are shown with thick (red) lines.

than 0.1 of the continuum intensity, we were left with a final line mask comprising 4177 lines, of these 1961 lines belong to Fe-peak elements, and 2122 lines belong to rare earth elements. The final line mask is dominated by Fe II (861 lines) and Ce II (977 lines).

The multiprofile LSD technique was applied to all observations of HD 125248 to obtain LSD profiles of Fe-peak and rare earth elements (Fig. 4). In addition, we computed mean LSD profiles for the entire mask. All LSD profiles were calculated with the following normalization coefficients: $\lambda_{0}=5000 \AA$, $d_{0}=1$, and $\bar{g}_{0}=1$.

The resulting LSD profiles for the Fe-peak and rare earth elements (Fig. 4) were compared to the profiles of several individual lines. This comparison showed that the resulting mean profiles properly describe the behavior with rotational phase of spectral lines of these species. The visual analysis of the LSD profiles shows what appears to be an almost total absence of rare earth element lines for phases around 0.0 when the lines of Fe-peak elements are strongest. Interpretation of this unusual variation with rotational phase of individual lines of Fe-peak and rare earth elements is the goal of our magnetic Doppler imaging analysis, which is presented in Sect. 5. 
Table 4. Integral magnetic field measurements obtained from the LSD Stokes profiles of HD 125248.

\begin{tabular}{|c|c|c|c|c|c|c|c|c|c|c|c|c|c|}
\hline \multirow[b]{2}{*}{$\operatorname{HJD}(2455000+)$} & \multirow[b]{2}{*}{ Phase } & \multicolumn{3}{|c|}{$\left\langle B_{\mathrm{Z}}\right\rangle \pm \sigma_{\left\langle B_{\mathrm{Z}}\right\rangle}(\mathrm{G})$} & \multicolumn{3}{|c|}{ null $\left\langle B_{\mathrm{Z}}\right\rangle \pm \sigma_{\left\langle B_{\mathrm{z}}\right\rangle}(\mathrm{G})$} & \multicolumn{3}{|c|}{$P_{Q} \pm \sigma_{P_{Q}}\left(\times 10^{-4}\right)$} & \multicolumn{3}{|c|}{$P_{U} \pm \sigma_{P_{U}}\left(\times 10^{-4}\right)$} \\
\hline & & full & Fe-peak & REE & full & Fe-peak & REE & full & $\mathrm{Fe}$-peak & REE & full & Fe-peak & REE \\
\hline 1017.77255 & 0.023 & $-1854 \pm 25$ & $-1811 \pm 20$ & $-553 \pm 164$ & $-5 \pm 4$ & $-6 \pm 4$ & $-4 \pm 30$ & $-1.5 \pm 1.1$ & $0.3 \pm 0.9$ & $-15.3 \pm 8.4$ & $8.8 \pm 1.1$ & $5.0 \pm 0.8$ & $24.0 \pm 7.6$ \\
\hline 1018.81486 & 0.136 & $-1052 \pm 24$ & $-1180 \pm 19$ & $893 \pm 116$ & $-6 \pm 5$ & $-8 \pm 4$ & $-2 \pm 27$ & $19.7 \pm 1.0$ & $21.4 \pm 0.8$ & $-8.7 \pm 5.7$ & $-12.7 \pm 1.0$ & $-8.1 \pm 0.8$ & $-33.1 \pm 5.3$ \\
\hline 1019.77340 & 0.239 & $571 \pm 20$ & $260 \pm 17$ & $1946 \pm 64$ & $-11 \pm 5$ & $-12 \pm 4$ & $-7 \pm 16$ & $2.8 \pm 1.1$ & $13.2 \pm 0.9$ & $-36.1 \pm 3.9$ & $-31.2 \pm 1.4$ & $-38.0 \pm 1.2$ & $7.0 \pm 4.8$ \\
\hline 1345.84686 & 0.317 & $1612 \pm 23$ & $1307 \pm 23$ & $2249 \pm 56$ & $-2 \pm 6$ & $11 \pm 6$ & $-28 \pm 15$ & $2.3 \pm 1.6$ & $-8.2 \pm 1.5$ & $35.3 \pm 4.1$ & $-22.1 \pm 1.6$ & $-24.9 \pm 1.5$ & $-10.3 \pm 4.0$ \\
\hline 1346.85386 & 0.425 & $1970 \pm 30$ & $1584 \pm 33$ & $2579 \pm 61$ & $-8 \pm 6$ & $-5 \pm 6$ & $-22 \pm 11$ & $9.8 \pm 2.1$ & $-8.4 \pm 2.2$ & $45.0 \pm 4.4$ & $17.7 \pm 1.6$ & $12.9 \pm 1.7$ & $26.2 \pm 3.4$ \\
\hline 1347.85514 & 0.533 & $1917 \pm 28$ & $1560 \pm 31$ & $2670 \pm 58$ & $6 \pm 5$ & $0 \pm 5$ & $13 \pm 10$ & $-17.1 \pm 1.3$ & $-13.4 \pm 1.3$ & $-24.2 \pm 2.7$ & $9.9 \pm 1.4$ & $6.0 \pm 1.4$ & $15.5 \pm 2.9$ \\
\hline 1348.86017 & 0.641 & $1497 \pm 21$ & $1223 \pm 21$ & $2256 \pm 55$ & $11 \pm 4$ & $13 \pm 4$ & $18 \pm 11$ & $-14.9 \pm 1.2$ & $-16.0 \pm 1.1$ & $-13.2 \pm 3.2$ & $11.7 \pm 1.2$ & $0.8 \pm 1.2$ & $42.5 \pm 3.3$ \\
\hline 1014.79860 & 0.704 & $807 \pm 19$ & $529 \pm 17$ & $1815 \pm 59$ & $14 \pm 4$ & $15 \pm 4$ & $11 \pm 12$ & $-20.2 \pm 1.1$ & $-15.6 \pm 1.0$ & $-36.9 \pm 3.9$ & $3.1 \pm 1.1$ & $15.7 \pm 1.0$ & $-43.0 \pm 4.0$ \\
\hline 1349.86061 & 0.749 & $135 \pm 20$ & $-111 \pm 16$ & $1371 \pm 76$ & $12 \pm 4$ & $17 \pm 4$ & $-30 \pm 18$ & $-23.4 \pm 1.1$ & $-20.2 \pm 0.9$ & $-35.6 \pm 4.6$ & $6.7 \pm 1.0$ & $12.3 \pm 0.8$ & $-22.7 \pm 4.2$ \\
\hline 1015.77181 & 0.808 & $-780 \pm 23$ & $-958 \pm 18$ & $601 \pm 106$ & $18 \pm 5$ & $17 \pm 4$ & $15 \pm 24$ & $-26.7 \pm 1.2$ & $-21.3 \pm 1.0$ & $-52.6 \pm 6.3$ & $14.3 \pm 1.0$ & $9.8 \pm 0.8$ & $39.5 \pm 5.4$ \\
\hline 1350.85141 & 0.855 & $-1339 \pm 24$ & $-1428 \pm 19$ & $-32 \pm 130$ & $15 \pm 4$ & $9 \pm 4$ & $58 \pm 24$ & $-16.7 \pm 1.1$ & $-12.1 \pm 0.8$ & $-39.2 \pm 6.5$ & $-0.1 \pm 1.0$ & $10.3 \pm 0.8$ & $-74.7 \pm 6.1$ \\
\hline 1016.80012 & 0.919 & $-1794 \pm 25$ & $-1794 \pm 19$ & $-523 \pm 155$ & $-4 \pm 4$ & $-2 \pm 3$ & $-27 \pm 25$ & $-12.4 \pm 1.0$ & $-12.0 \pm 0.8$ & $-5.5 \pm 7.2$ & $15.2 \pm 1.1$ & $10.3 \pm 0.8$ & $49.4 \pm 7.5$ \\
\hline
\end{tabular}

Notes. First and second columns list heliocentric JD and rotational phase, calculated according to the improved period (Sect. 4.1). Columns 35 provide $\left\langle B_{\mathrm{z}}\right\rangle$ measurements for the Stokes $V$ LSD profiles obtained with the full mask, for the lines of Fe-peak elements, and for the REE lines. Columns 6-8 provide the $\left\langle B_{\mathrm{z}}\right\rangle$ measurements from the null Stokes $V$ profiles. Columns 9-11 report the Stokes $Q$ net linear polarization measurements for the same three sets of LSD profiles, and Cols. 12-14 provide the net linear polarization measurements for the LSD Stokes $U$ profiles.

We used the LSD profiles and calculated the longitudinal magnetic field, $\left\langle B_{\mathrm{z}}\right\rangle$, and net linear polarization $P_{Q}$ and $P_{U}$. The former is a useful measurement of the line-of-sight component of the magnetic field. It can be calculated from the first order moment of the Stokes $V$ profiles (Kochukhov et al. 2010). This is the most commonly used magnetic observable for early-type stars. The net linear polarization is calculated from the equivalent width of the LSD Stokes $Q$ and $U$ profiles and contains information about the transverse component of the magnetic field that is similar to what is diagnosed by the broadband linear polarization (Leroy 1995). However, direct comparison between the two types of measurements is not possible unless they are scaled and shifted (e.g., Wade et al. 2000; Rusomarov et al. 2013).

The longitudinal field and net linear polarization measurements are presented in Table 4. The calculations were done for Fe-peak, rare earth elements, and for the LSD profiles obtained with the full mask. The measurement uncertainties were obtained from the actual LSD profiles using the standard error propagation principle. In the same way we calculated the equivalent quantities from the null profiles, which were derived by applying the line mask and the LSD technique to the null Stokes spectra. These quantities are excellent indicators of spurious polarization signals. Our analysis of these quantities showed that any spurious contributions to the measurements presented in this section are well below the uncertainties due to the finite signalto-noise ratio of the observations.

The full LSD line mask was also applied to the circular polarization measurements obtained at the NOT. We use the resulting mean longitudinal field measurements only to extend the baseline for the rotational period determination in Sect. 4.1. The $\left\langle B_{z}\right\rangle$ measurements from SOFIN spectra are presented in Table 5.

\subsection{Rotational period}

Given a large number of magnetic field studies of HD 125248 it is possible to find a highly precise rotational period. The analyzed list of longitudinal magnetic field measurements contains all published data with the exception of measurements produced only from rare earth element lines. We also did not include the early measurements by Babcock (1951) as they appeared to be too inaccurate for our study. The data by Babcock (1958) were also discarded as this author did not provide accurate enough observation dates. Our list of $\left\langle B_{\mathrm{z}}\right\rangle$ measurements includes the Fe I, Fe II, and Ti II estimates by Hockey (1969), measurements of Fe II lines by Mathys (1994) and Mathys \& Hubrig (1997), and
Table 5. Mean longitudinal magnetic field of HD 125248 obtained from NOT observations.

\begin{tabular}{ccr}
\hline HJD & Phase & $\left\langle B_{\mathrm{z}}\right\rangle(\mathrm{G})$ \\
\hline 2452064.44814 & 0.733 & $274 \pm 108$ \\
2452065.46507 & 0.842 & $-1316 \pm 122$ \\
2452066.46713 & 0.950 & $-2125 \pm 125$ \\
2452067.46349 & 0.057 & $-1900 \pm 121$ \\
2452068.47056 & 0.165 & $-698 \pm 107$ \\
2452069.46535 & 0.272 & $1144 \pm 108$ \\
2452070.46415 & 0.380 & $1940 \pm 143$ \\
2452071.46090 & 0.487 & $2110 \pm 157$ \\
2452072.44919 & 0.593 & $1815 \pm 132$ \\
\hline
\end{tabular}

Notes. These measurements were derived from the LSD profiles calculated using the full mask.

data by Leone \& Catanzaro (2001) and Shorlin et al. (2002). To this list we also added the photopolarimetric longitudinal field measurements from the wings of $\mathrm{H} \beta$ lines by Landstreet et al. (1975) and Borra \& Landstreet (1980). However, we rescaled these measurements by a factor of 0.85 to better match the observed $\left\langle B_{z}\right\rangle$ amplitude from the Fe-peak elements. These data were finally complemented by our own longitudinal field measurements from the recent HARPSpol observations and older NOT observations.

We performed period search on the entire data set, comprised of 78 measurements in total, spanning over a time period of more than 40 years. As a starting guess for the fitting procedure we used the last reported period, $P_{\text {rot }}=9.29545 \mathrm{~d}$, derived by Leone \& Catanzaro (2001), and adopted the zero point from their paper. The best-fit curve was derived using four frequency components, which is necessary to reproduce the observed variations of the longitudinal field measurements. With the zero point from Leone \& Catanzaro (2001) and our improved stellar rotational period, the final ephemeris is given by

$$
\operatorname{HJD}\left(\left\langle B_{\mathrm{z}}\right\rangle_{\min }\right)=2433103.95+9.29558(6) \cdot E .
$$

Figure 5 shows the variations of the mean longitudinal field measurements of HD 125248 phased according to the revised rotational period. We computed all phases according to the ephemeris given above. 


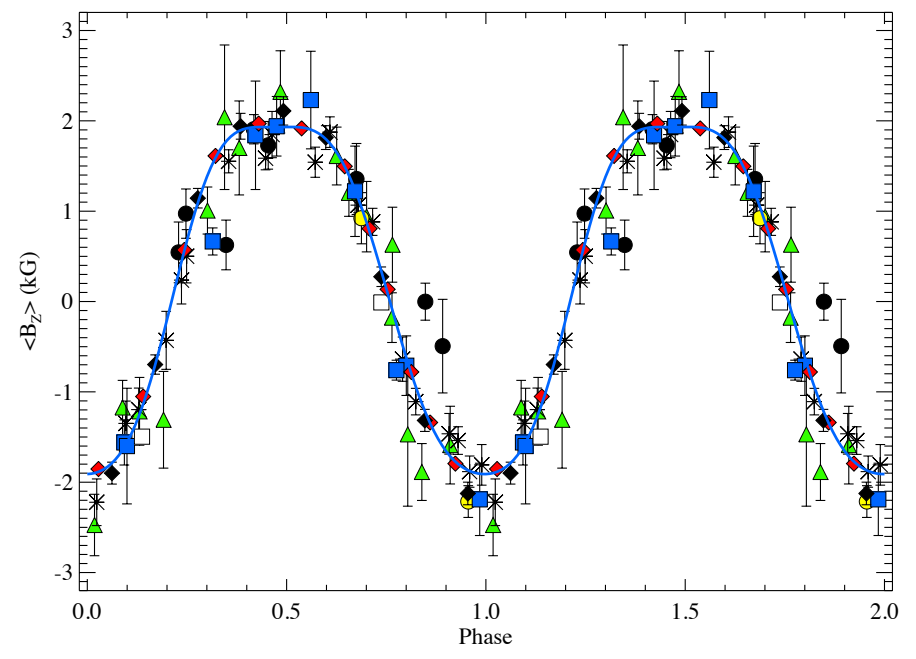

Fig. 5. Variability of the longitudinal magnetic field of HD 125248 with rotational phase. The symbols correspond to the following data sets: HARPSpol (red diamonds), NOT (black diamonds), Hockey (1969, black bullets), Landstreet et al. (1975, green triangles), Borra \& Landstreet (1980, green triangles), Mathys (1994, asteriks), Mathys \& Hubrig (1997, yellow bullets), Leone \& Catanzaro (2001, blue squares), Shorlin et al. (2002, white squares). The solid thick curve is a fourth-order Fourier fit to the longitudinal field measurements.

\section{Magnetic Doppler imaging}

\subsection{Methodology}

The availability of high-quality spectropolarimetric observations in all four Stokes parameters for HD 125248 gives us the opportunity to carry out a detailed tomographic reconstruction of its magnetic field and chemical abundance distributions. Magnetic Doppler imaging (MDI) is a computational technique that attempts to fit a set of observed Stokes parameters with synthetic spectra by adjusting the surface distribution of the magnetic field and the abundance of one or more chemical elements. The surface distribution maps are adjusted iteratively until the synthetic spectra properly describe the behavior of the observed line profiles with rotational phase in all Stokes parameters. We performed this task with the MDI code INVERS10 introduced by Piskunov \& Kochukhov (2002) and Kochukhov \& Piskunov (2002). In this study, we closely follow already established methodological practices that have been used in recent MDI studies (e.g., Rusomarov et al. 2015; Kochukhov et al. 2015).

In the case when we have a full Stokes $I Q U V$ vector of spectropolarimetric observations, we can find the surface abundance $\varepsilon$ and magnetic field distribution $\boldsymbol{B}$ maps by solving the following nonlinear least-squares minimization problem:

$$
\begin{aligned}
\Psi(\varepsilon, \boldsymbol{B})= & \sum_{\varphi \lambda} w_{I}\left(I_{\varphi \lambda}^{\mathrm{obs}}-I_{\varphi \lambda}^{\mathrm{calc}}(\varepsilon, \boldsymbol{B})\right)^{2} / \sigma_{I \varphi \lambda}^{2} \\
& +\sum_{\varphi \lambda} w_{Q}\left(Q_{\varphi \lambda}^{\mathrm{obs}}-Q_{\varphi \lambda}^{\mathrm{calc}}(\varepsilon, \boldsymbol{B})\right)^{2} / \sigma_{Q \varphi \lambda}^{2} \\
& +\sum_{\varphi \lambda} w_{U}\left(U_{\varphi \lambda}^{\mathrm{obs}}-U_{\varphi \lambda}^{\mathrm{calc}}(\varepsilon, \boldsymbol{B})\right)^{2} / \sigma_{U \varphi \lambda}^{2} \\
& +\sum_{\varphi \lambda} w_{V}\left(V_{\varphi \lambda}^{\mathrm{obs}}-V_{\varphi \lambda}^{\mathrm{calc}}(\varepsilon, \boldsymbol{B})\right)^{2} / \sigma_{V \varphi \lambda}^{2} \\
& +\Lambda_{a} \cdot \mathcal{R}_{a}(\varepsilon)+\Lambda_{f} \cdot \mathcal{R}_{f}(\boldsymbol{B}) \rightarrow \min ,
\end{aligned}
$$

where $\lambda$ and $\varphi$ are the wavelength and rotational phase of each spectral pixel and $\sigma_{\lambda \varphi}$ denotes its relative error. The functions $\mathcal{R}_{a}$ and $\mathcal{R}_{f}$ are the regularization functionals of the abundance and magnetic field distribution maps and $\Lambda_{a}$ and $\Lambda_{f}$ are the respective regularization parameters. To ensure that the relative contribution to the total discrepancy function $\Psi$ of different Stokes parameters is comparable, we introduce the weights $w$. The parameters $\Lambda_{a}$ and $\Lambda_{f}$ control the contribution of the regularization that is included into the total discrepancy function $\Psi$. In practice we do not allow the contribution of the regularization to become less than 10 percent of the total discrepancy function. This ensures that our maps reproduce the observations reasonably well without fitting them down to the noise level.

In this study we use first order Tikhonov regularization (Tikhonov \& Arsenin 1977) for the abundance maps and a penalty function of the form $\sum_{\ell, m} \ell^{2}\left(\alpha_{\ell m}+\beta_{\ell m}+\gamma_{\ell m}\right)^{2}$ for the magnetic field (Donati et al. 2006; Kochukhov et al. 2014). The Tikhonov regularization leads to the smoothest possible maps for the abundance distributions as permitted by the observations; the penalty function constrains the inversion so that the magnetic field map contains the least possible contribution from highorder harmonic modes.

Special care needs to be taken when computing the synthetic spectra in Eq. (1). We calculate the local Stokes profiles for a large number of points on the stellar surface to model the polarization signatures in a realistic way. We then convolve these profiles with a Gaussian to simulate the spectral resolution of the instrument and Doppler shift the resulting profiles for each rotational phase $\varphi$. The local profiles are then integrated over the visible stellar disk for each phase $\varphi$ and wavelength point $\lambda$. Finally they are normalized by a phase-independent continuum intensity. The integration procedure in INVERS 10 also accounts for the projected area of each surface element for each phase $\varphi$.

An important characteristic of the INVERS10 code is the way we compute the local Stokes profiles. Instead of using simplifying approximations in the form of fixed local Gaussian profiles or Milne-Eddington atmospheres (e.g., Donati \& Brown 1997), INVERS10 solves the equations of polarized radiative transfer numerically in a realistic model atmosphere for each point on the stellar surface.

The version of the INVERS10 code used here represents the magnetic field as a superposition of poloidal and toroidal components, each expressed as a spherical harmonics series (Donati et al. 2006; Kochukhov et al. 2014). In this formalism, the field is represented by a set of harmonic coefficients $\alpha_{\ell m}$, $\beta_{\ell m}$, and $\gamma_{\ell m}$, representing the poloidal radial, poloidal horizontal, and toroidal horizontal components, respectively. The expansion of the magnetic field components is truncated at $\ell_{\max }$. For HD 125248, given the relatively low projected rotational velocity, we found that an expansion up to $\ell_{\max }=10$ is sufficient to describe the magnetic field.

\subsection{Spectral line selection}

Simultaneous reconstruction of the surface magnetic field and abundance distributions of as many chemical elements as possible facilitates the achievement of a robust MDI solution (Kochukhov \& Piskunov 2002; Silvester et al. 2014a). However, these two goals have slightly different requirements when it comes to line selection. The strong magnetically sensitive lines are excellent for magnetic field mapping, but they are not optimal for abundance reconstruction because they may be saturated over a large area of the stellar surface. In contrast, the weaker lines are better suited for abundance mapping and for deriving the projected rotational velocity $v_{\mathrm{e}} \sin i$, but owing to the low amplitude of polarization signals, are less suitable for magnetic 
Table 6. Spectral lines used in magnetic Doppler imaging of HD 125248 .

\begin{tabular}{lcccc}
\hline \hline Ion & $\lambda(\AA)$ & $E_{i}(\mathrm{eV})$ & $\log g f$ & $\bar{g}$ \\
\hline Ce II & 4460.207 & 0.478 & 0.280 & 0.690 \\
Ce II & 4486.909 & 0.295 & -0.180 & 0.985 \\
Ce II & 4562.359 & 0.478 & 0.210 & 1.028 \\
\hline Gd II & 4597.910 & 0.602 & -0.830 & 1.585 \\
Gd II & 4732.609 & 1.102 & -0.540 & 1.555 \\
Gd II & 5092.249 & 1.727 & -0.230 & 1.535 \\
\hline Nd III & 5677.179 & 0.631 & -1.450 & 1.600 \\
Nd III & 5851.542 & 0.461 & -1.550 & 1.660 \\
Nd III & 6145.068 & 0.296 & -1.330 & 0.690 \\
\hline Cr II & 4554.988 & 4.071 & -1.282 & 1.330 \\
Cr II & 4618.803 & 4.074 & -0.840 & 0.918 \\
Cr II & 4824.127 & 3.871 & -0.970 & 1.340 \\
Cr II & 5420.922 & 3.758 & -2.458 & 1.490 \\
Cr II & 6134.465 & 6.578 & $-2.285^{*}$ & 0.595 \\
Cr II & 6138.721 & 6.484 & $-1.793^{*}$ & 0.915 \\
Cr II & 6336.263 & 4.073 & -3.759 & 0.665 \\
\hline Fe II & 4508.280 & 2.856 & -2.250 & 0.500 \\
Fe II & 4576.333 & 2.844 & -2.920 & 1.185 \\
Fe II & 5018.436 & 2.891 & -1.220 & 1.935 \\
Fe II & 5061.703 & 10.308 & $0.606^{*}$ & 1.360 \\
Fe II & 5169.028 & 2.891 & -1.250 & 1.325 \\
Fe II & 5197.568 & 3.230 & -2.100 & 0.660 \\
Fe I & 5615.644 & 3.332 & $-0.050^{*}$ & 1.200 \\
Fe II & 5961.706 & 10.678 & $0.995^{*}$ & 1.192 \\
Fe II & 5991.371 & 3.153 & -3.540 & 0.813 \\
Fe II & 6084.102 & 3.199 & $-3.930^{*}$ & 0.712 \\
Fe II & 6446.407 & 6.223 & -1.960 & 1.240 \\
Fe II & 6482.204 & 6.219 & $-1.803^{*}$ & 0.940 \\
\hline & & & &
\end{tabular}

Notes. The columns give the ion, central wavelength $\lambda$, excitation potential of the lower atomic level $E_{i}$, the oscillator strength $\log g f$, and the effective Landé factor $\bar{g}$ of the spectral lines used for the inversions. ${ }^{(*)} \log g f$ value automatically adjusted in the MDI inversion.

field mapping if used by themselves. Therefore, a mix of lines with different magnetic sensitivity and line strength is necessary to achieve a robust reconstruction of the surface magnetic field and abundance distributions. An additional line selection criterion is also the absence of significant blending.

On the basis of these criteria, we selected $12 \mathrm{Fe} \mathrm{I/II,} 7 \mathrm{Cr}$ II, and three lines each for Ce II, Gd II, and Nd III ions. Some of the Fe and Cr lines, for example, Fe II $\lambda 5018 \AA$, Cr II $\lambda 4824 \AA$, as well as Nd III $\lambda 5851 \AA$ have already been used in previous MDI studies. Unfortunately, other well-known Fe II lines with strong polarization signals are severely blended by other elements in the spectrum of HD 125248 and could not be used in our MDI study. The line selection contains lines with excitation energy spanning a range of close to $10 \mathrm{eV}$, and $\log g f$ factors that span $3.4 \mathrm{dex}$. We used lines that have low magnetic sensitivity $(\bar{g} \leq 1.0)$ and lines that are strongly sensitive with large Lande factors $(\bar{g} \geq 1.5)$.

The line list adopted for the MDI of HD 125248 is presented in Table 6. The atomic data for these lines were extracted from the latest version of the VALD database (Ryabchikova et al. 2015). As reported in Sect. 3.1, we found a definite trend between the relative strength and abundance for spectral lines of the Fe II and CrII ions. Such discrepancies have also been reported for other magnetic Ap stars and are linked to vertical stratification (Ryabchikova et al. 2005; Ryabchikova 2014). Part of these discrepancies may be caused by non-LTE effects. Since neither non-LTE effects nor vertical chemical stratification is included in our MDI modeling we introduced additional corrections to the oscillator strengths of certain lines. These corrections were automatically calculated by the INVERS 10 code.

\subsection{Optimization of $v_{\mathrm{e}} \sin i$, and orientation of the rotation axis}

In addition to an accurate stellar atmosphere model and a complete line list, MDI also requires knowledge of the orientation of the stellar rotation axis and the projected rotational velocity $v_{\mathrm{e}} \sin i$. The orientation of the rotation axis is specified by the two angles $i$ and $\Theta$. The inclination $i$ is the tilt of the rotation axis relative to the observer's line of sight; this inclination has values in the range $\left[0^{\circ}, 180^{\circ}\right]$. The values of $i$ between $0^{\circ}$ and $90^{\circ}$ correspond to the situation when the star rotates counterclockwise as seen by the observer. The azimuth angle $\Theta$ determines the orientation of the rotation axis in the plane on the sky; this angle has values in the range $\left[0^{\circ}, 360^{\circ}\right]$. However, because the Stokes $Q U$ parameters depend on the trigonometric functions of $2 \Theta$, there is an inherent ambiguity between values of $\Theta$ and $180^{\circ}+\Theta$.

Kochukhov \& Piskunov (2002) showed that incorrect values of these parameters lead to increase of the $\chi^{2}$ value for the fit to the observed Stokes profiles. An incorrect projected rotational velocity $v_{\mathrm{e}} \sin i$ leads to worse fit for the Stokes $I$ parameters; incorrect values for the $i$ and $\Theta$ parameters results in similarly worse fit for the Stokes $Q$ and $U$ parameters.

The initial value for the inclination $i=75^{\circ} \pm 25^{\circ}$ (or $i=105^{\circ} \pm 25^{\circ}$ ) was calculated from the oblique rotator relation taking into account the stellar radius $R=2.23 R_{\odot}$ and the projected rotational velocity $v_{\mathrm{e}} \sin i=11.5 \pm 1.5 \mathrm{~km} \mathrm{~s}^{-1}$ estimated in Sect. 3, and the rotational period $P_{\text {rot }}=9.29558 \mathrm{~d}$ found in Sect. 4.1.

The initial guess for the azimuthal angle $\Theta$ of the rotational axis was estimated in the following way. We fitted our longitudinal magnetic field and net linear polarization measurements calculated for the full mask (Sect. 4, Table 4) with predictions of the "canonical" model for a dipolar field geometry (Landolfi et al. 1993). We did not rescale the net linear polarization measurements to match the observed amplitude of the broadband linear polarization measurements (Leroy 1995) because there are only five such measurements for HD 125248 with error bars comparable to the amplitude of the observations themselves. Besides, we only aimed to produce a rough estimate of the azimuthal angle $\Theta$ and investigate how it depends on $i$, keeping in mind that a dipole model is known to produce worse results than a more sophisticated combination of a dipole and quadrupole models (Bagnulo et al. 1999). With these considerations we found that for different values of $i=75^{\circ} \pm 25^{\circ}$ the azimuthal angle was always close to $30^{\circ}$. This is the value that we adopted as an initial guess.

We then optimized the values of $i$ and $\Theta$ by calculating $81 \mathrm{MDI}$ inversions on a grid $i \in\left[35^{\circ}, 115^{\circ}\right]$ and $\Theta \in\left[0^{\circ}, 80^{\circ}\right]$ with $10^{\circ}$ steps for both angles. The minimum value of $\chi^{2}$ for the fit to the Stokes $Q$ and $U$ profiles when considering all spectral lines was found at $i=95^{\circ}$ and $\Theta=20^{\circ}$. Furthermore, we inspected the position of the minima separately for $\mathrm{Fe}$ and $\mathrm{Cr}$ and the rare earth elements. We found out that the minimum for the combined set of $\mathrm{Fe}$ and $\mathrm{Cr}$ lines is close to that derived for all lines. The rare earth element lines have minimum at $i=105^{\circ}$ and $\Theta=40^{\circ}$. Because we are simultaneously modeling both groups of lines, we adopted $i=95^{\circ}$ and $\Theta=30^{\circ}$ and $10^{\circ}$ errors for both 


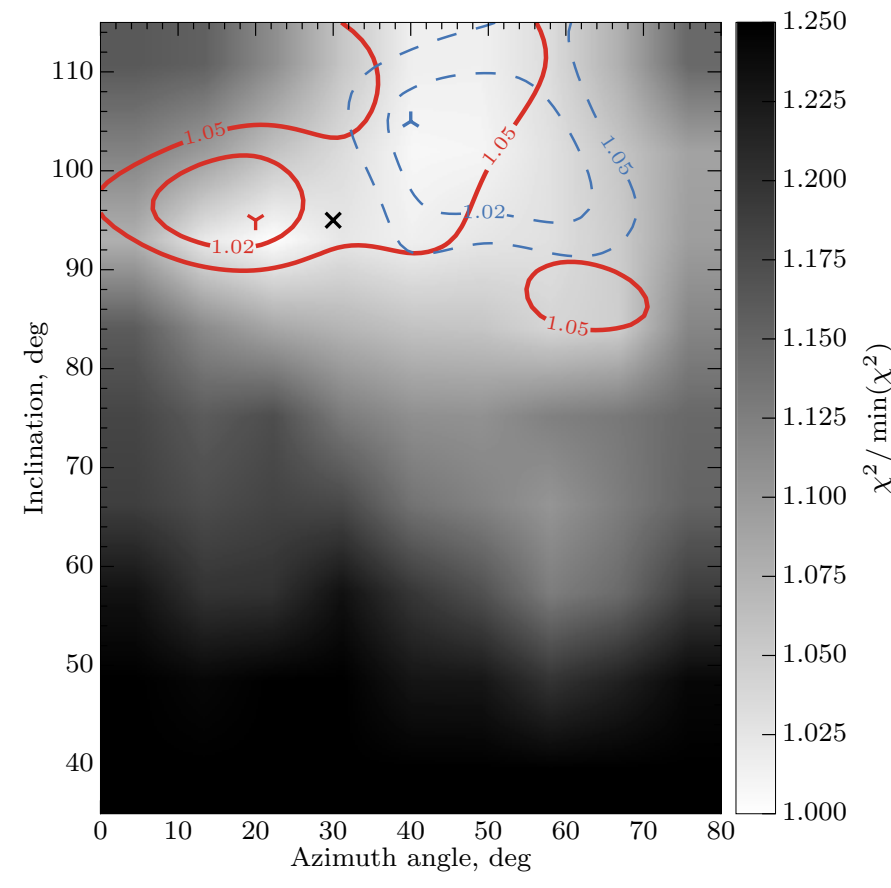

Fig. 6. Variation of $\chi^{2}$ of the fit to the Stokes $Q U$ profiles with inclination $i$ and azimuth angle $\Theta$. The contours plotted with thick (red) lines show the $2 \%$, and $5 \%$ increase from the minimum indicated with a $Y$ symbol for the $\mathrm{Fe}$ and $\mathrm{Cr}$ lines. The contours plotted with dashed (blue) lines show the same for the rare earth lines, the minimum in this case is indicated with a $\curlywedge$ symbol. The $\times$ symbol indicates the adopted values of $i$ and $\Theta$.

quantities. The goodness of fit for the Stokes $Q U$ parameters is shown in Fig. 6.

We optimized the value of the projected rotational velocity in a similar manner. We carried out 13 MDI inversions with values of $v_{\mathrm{e}} \sin i$ in the range from $6 \mathrm{~km} \mathrm{~s}^{-1}$ to $19 \mathrm{~km} \mathrm{~s}^{-1}$. We then computed the $\chi^{2}$ for each value of $v_{\mathrm{e}} \sin i$ for the Stokes $I$ profiles of the $\mathrm{Fe}$ and $\mathrm{Cr}$ lines and the rare earth elements lines. These results are illustrated in Fig. 7. We found that both groups favor $v_{\mathrm{e}} \sin i$, which is very close to that derived in the abundance analysis of the average spectrum (Sect. 3.1). The minimum for the $\mathrm{Fe}$ and $\mathrm{Cr}$ lines is located at $11.4 \mathrm{~km} \mathrm{~s}^{-1}$, while the rare earth element lines indicate a value close to $11.1 \mathrm{~km} \mathrm{~s}^{-1}$. Using the solid body rotation relation and the newly derived value for the inclination, we found that $v_{\mathrm{e}} \sin i=11.4 \mathrm{~km} \mathrm{~s}^{-1}$ leads to $R=2.1 R_{\odot}$, while the value derived from the rare earth element lines yields $R=2.0 R_{\odot}$. Both values are consistent with $R$ derived from SED fitting in Sect. 3. Based on these results, we adopted $v_{\mathrm{e}} \sin i=11.4 \pm 0.5 \mathrm{~km} \mathrm{~s}^{-1}$ for the rest of the study.

\section{Magnetic field and chemical abundance distributions}

The magnetic field topology of HD 125248 was derived from the simultaneous mapping of $\mathrm{Fe}, \mathrm{Cr}, \mathrm{Ce}, \mathrm{Nd}$, and $\mathrm{Gd}$. We fitted the phase variations of the Stokes IQUV spectra for the line list presented in Table 6. In Fig. 8 we plot the spherical projection of the resulting radial field component, the field modulus, and the strength of the horizontal field components; the bottom row shows the vector magnetic field. The comparison of the observed and computed Stokes parameter spectra is presented in Figs. 9-12.

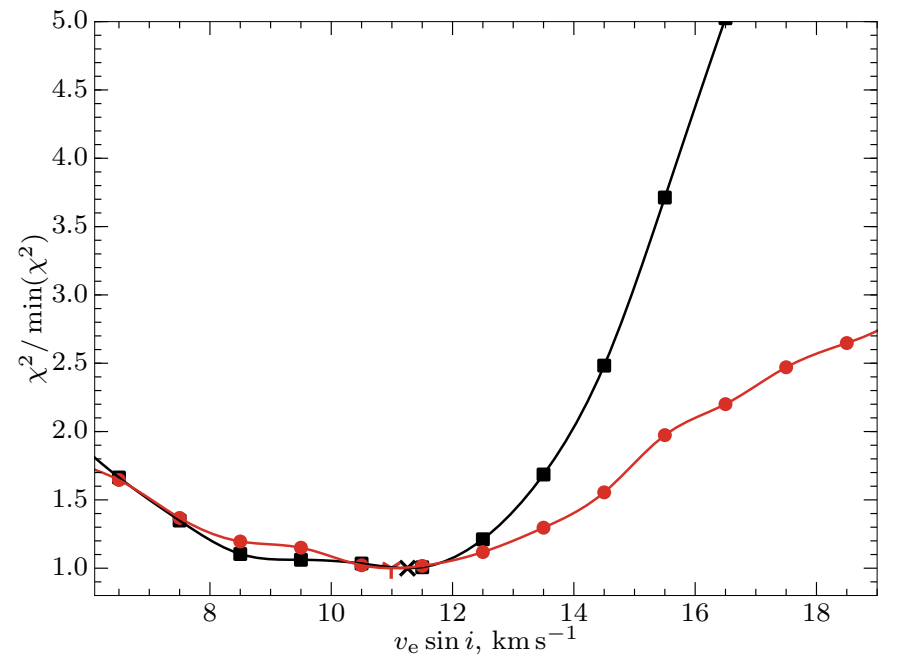

Fig. 7. Variation of $\chi^{2}$ of the fit to the Stokes $I$ profiles as a function of the projected rotational velocity. The curve, indicated with squares (black), shows the normalized deviation for the Fe and $\mathrm{Cr}$ lines; their minimum is indicated with the $\times$ symbol. The curve indicated with bullets (red) shows the same for the rare earth element lines; the minimum is shown with the $Y$ symbol. The solid curves are interpolating cubic splines used to find the optimal projected rotational velocity.

The MDI inversions indicate that the magnetic field structure of HD 125248 is dominated by two regions of different polarity. The area with negative polarity (inward oriented field vector) has a field modulus that is on average stronger by about $30 \%$ than the area with positive polarity (outward oriented field vector). We take this as evidence of a strong asymmetry between the field strength of the positive and negative magnetic poles. Furthermore, the area where the positive radial field reaches its maximum strength corresponds to an extended arc that almost wraps itself around the rotational pole. This can most easily be seen for the radial field component in Fig. 8 for phases 0.4 and 0.6. The area with the strongest positive radial field, as traced by the contours in the plot, forms a structure that is extended in the longitudinal direction, whereas the negative radial field area is much more symmetric and localized.

The difference in field strength between the regions of different polarity also manifests itself in their relative surface areas. This is a consequence of magnetic flux conservation, which is enforced by representing the field as a superposition of a poloidal and toroidal components in the inversion. The much stronger negative field encompasses an area that is approximately two times smaller than the surface area of the field with positive polarity.

The estimated disk-integrated mean field modulus of HD 125248 varies between 3.5 and $6 \mathrm{kG}$ with a large $3.5 \mathrm{kG}$ strength plateau between the rotational phases 0.3 and 0.7 . This estimate of the field modulus is somewhat smaller, but still in line with previous estimates by Glagolevskij (2007) and Bagnulo et al. (1999).

The analysis of the contribution of different harmonic components to the total energy of the field of HD 125248 showed that the field is mainly poloidal ( 71 percent of the total magnetic energy) with a dominant contribution from the poloidal dipolar component (53 percent of the total magnetic energy). The contribution of the toroidal components for all modes is relatively strong with 29 percent of the total magnetic energy of HD 125248. The quadrupolar $(\ell=2)$ and octupolar $(\ell=3)$ components contribute 27 percent of the total energy of the field. 

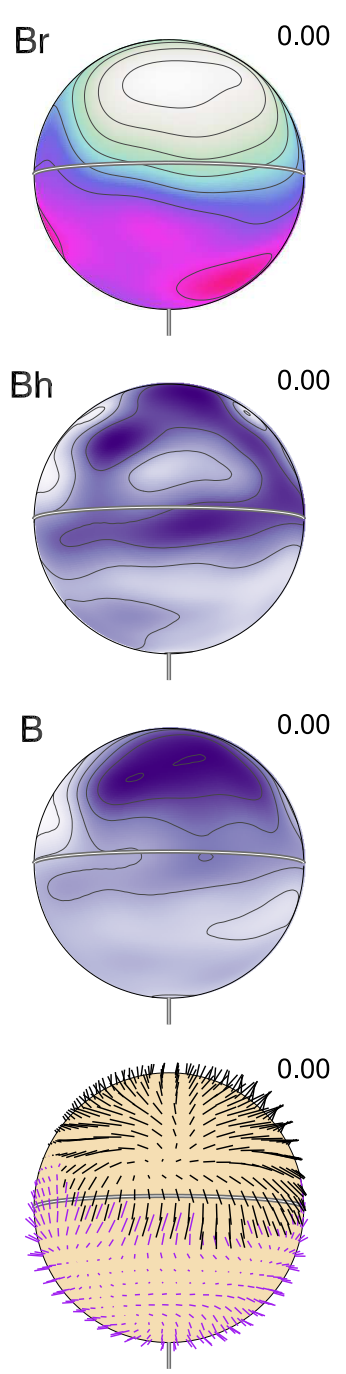

0.20

0.20
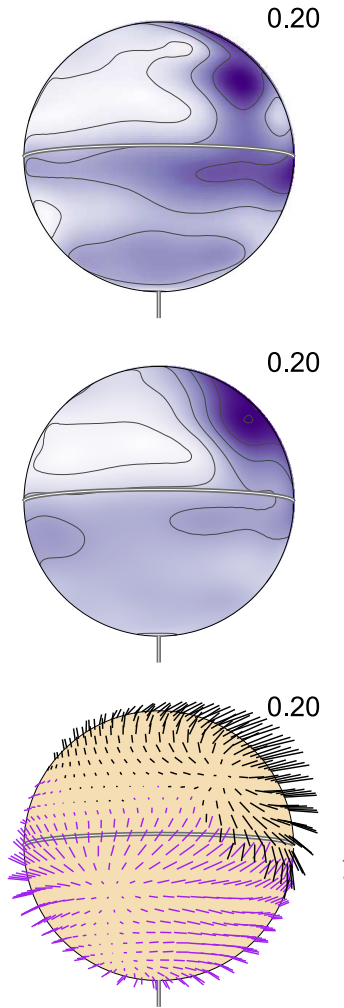

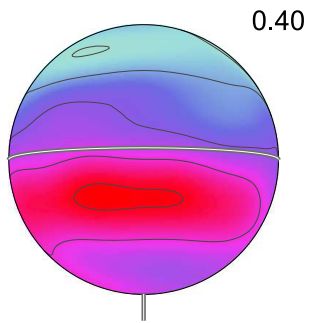

0.40

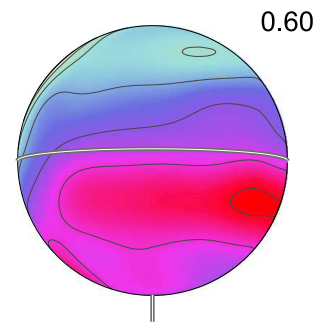

0.40
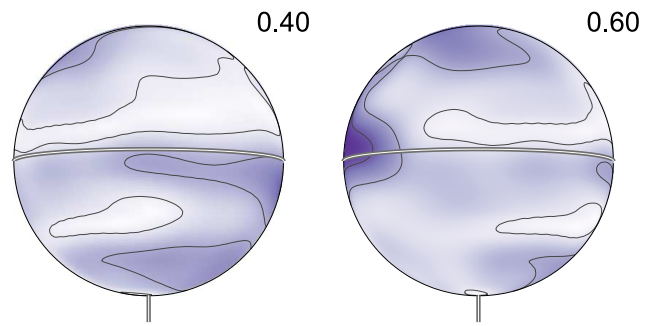

0.40
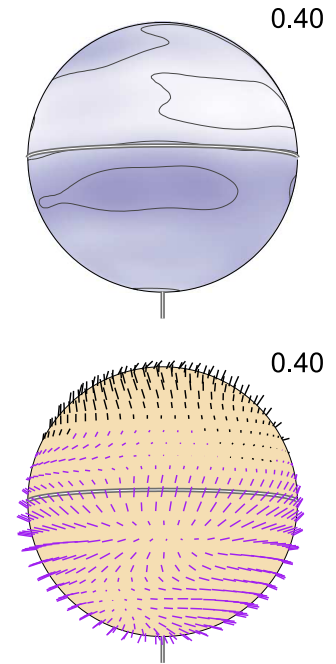

0.60

0.60
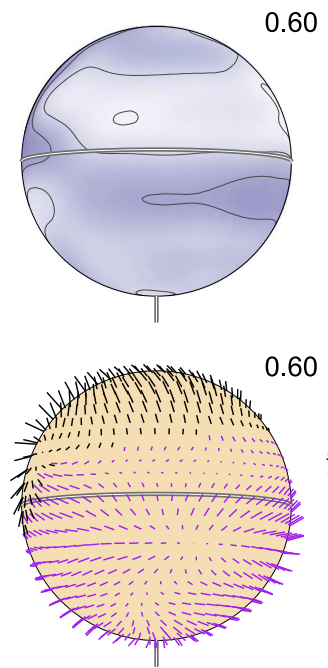
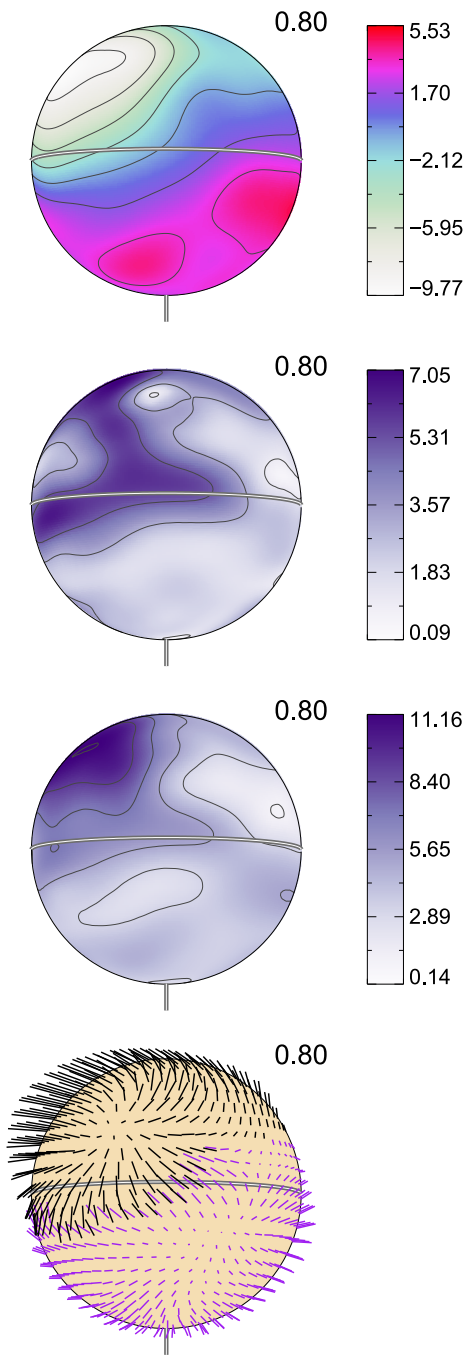

Fig. 8. Distribution of the magnetic field on the surface of HD 125248 derived from simultaneous MDI analysis of $\mathrm{Fe}, \mathrm{Cr}, \mathrm{Nd}, \mathrm{Ce}$, and $\mathrm{Gd}$. The plots show the distribution of magnetic radial field (first row), horizontal field (second row), field modulus (third row), and field orientation (fourth row). The color bars on the right indicate the field strength in $\mathrm{kG}$. The contours are plotted with $2 \mathrm{kG}$ steps. The arrow length in the bottom plot is proportional to the field strength. The star is shown at five rotational phases, indicated above each spherical plot. The thick line and the vertical bar indicate positions of the rotational equator and the pole respectively.

Higher order modes with $\ell \geq 4$ contribute less than 10 percent of the total field energy. Figure 13 compares the energies of the poloidal and toroidal components as a function of the angular degree $\ell$.

We carried out inversions in which we limited the maximum number of spherical harmonics for the field expansion to confirm that reproducing the observed Stokes profile variation indeed requires components with $\ell>2$. Our first test involved searching a best-fit dipole field $\left(\ell_{\max }=1\right)$; then we fitted the observed Stokes IQUV profiles with a combination of a dipole and quadrupole field topologies $\left(\ell_{\max }=2\right)$. Our best fits to the Stokes profiles are shown in Fig. 14. For the sake of brevity, we only plotted the fit for seven spectral lines, which are representative of the entire set. We carried out the inversions for the entire line list and we allowed the code to fit arbitrary abundance maps to the observed profiles.

It is evident that a dipole field cannot properly reproduce the observations. In some cases, it cannot even reproduce the width of certain lines for phases $\varphi \approx 0$ when the field strength is at its maximum, for example, Cr II $\lambda 5421 \AA$. The theoretical Stokes $V$ parameters are systematically underestimated for phases when the field is strongest. For several other lines the synthetic Stokes $V$ profiles have different morphology than the observed profiles. The $\chi^{2}$ of the fit to the Stokes IQUV profiles for the dipole field is larger by a factor of three compared to the inversion, which includes higher order harmonic components.

The dipole plus quadrupole fit on average reproduces the intensity spectra much better, keeping the theoretical Stokes $V$ profiles close to the observed profiles. Thus, one can indeed say that on the largest scales the field of HD 125248 is better represented by a combination of a dipole and quadrupole field. However, on closer inspection we can see that this model systematically fails to reproduce the Stokes $Q U$ profiles of most lines when the field is at its maximum, for example, Fe II $\lambda 5018 \AA$, Cr II $\lambda 5421 \AA$, and Cr II $\lambda 4824 \AA$. This suggests that the surface field structures introduced by the harmonics with $\ell \geq 3$ are statistically significant and justified by the observational data.

\subsection{Abundance maps}

We reconstructed the abundance distribution maps of six elements in total. The abundance maps of $\mathrm{Fe}, \mathrm{Cr}, \mathrm{Nd}, \mathrm{Ce}$, and $\mathrm{Gd}$ were obtained simultaneously with the magnetic field inversion. 


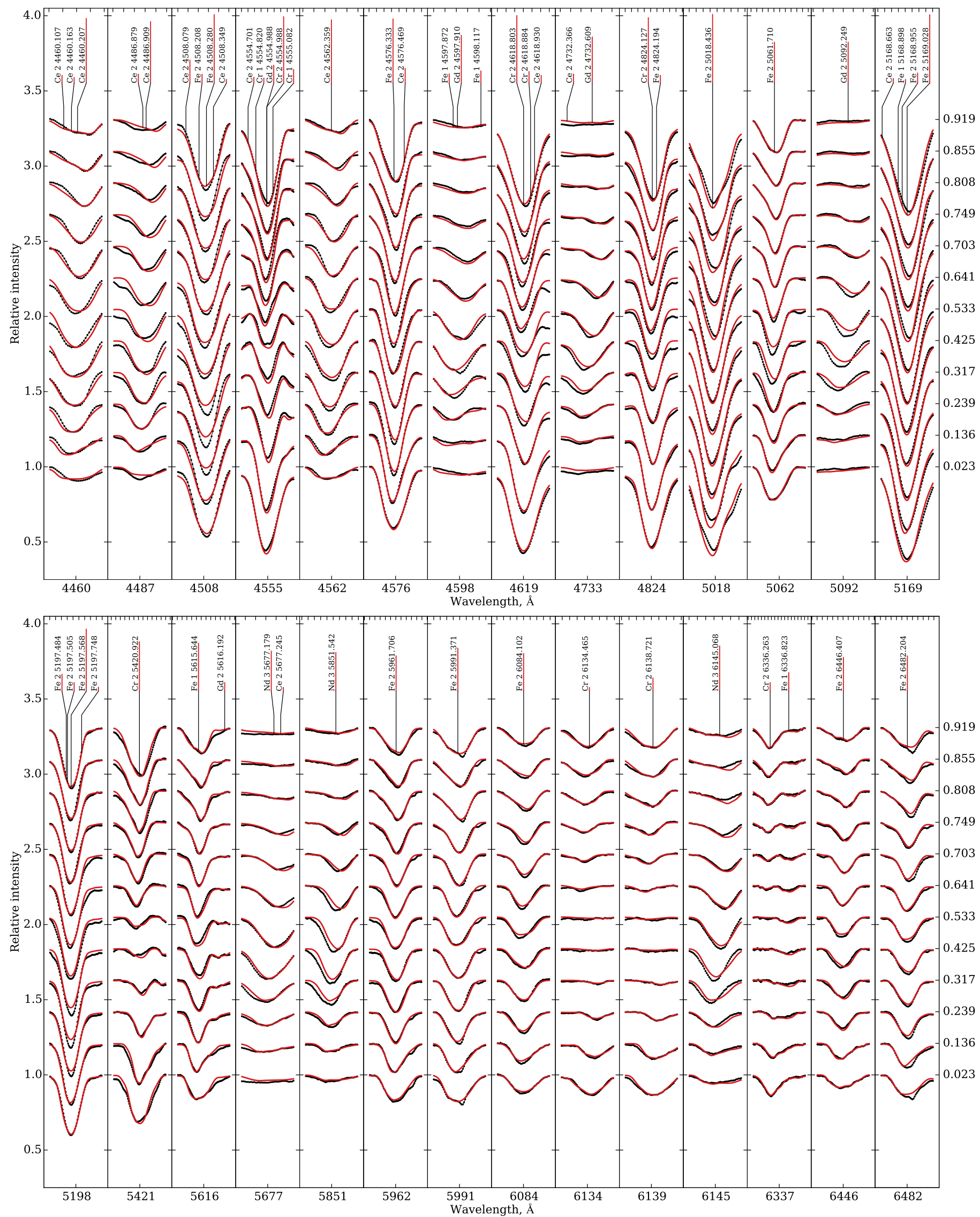

Fig. 9. Comparison of the observed (dots connected with black lines) and synthetic (thin red lines) Stokes I profiles calculated for the final magnetic field and abundance maps. The distance between two horizontal tick marks on the upper axis of each panel is $0.1 \AA$, indicating the wavelength scale. Rotational phases are given on the right of both panels. 
N. Rusomarov et al.: Magnetic field and abundance spots of the Ap star HD 125248

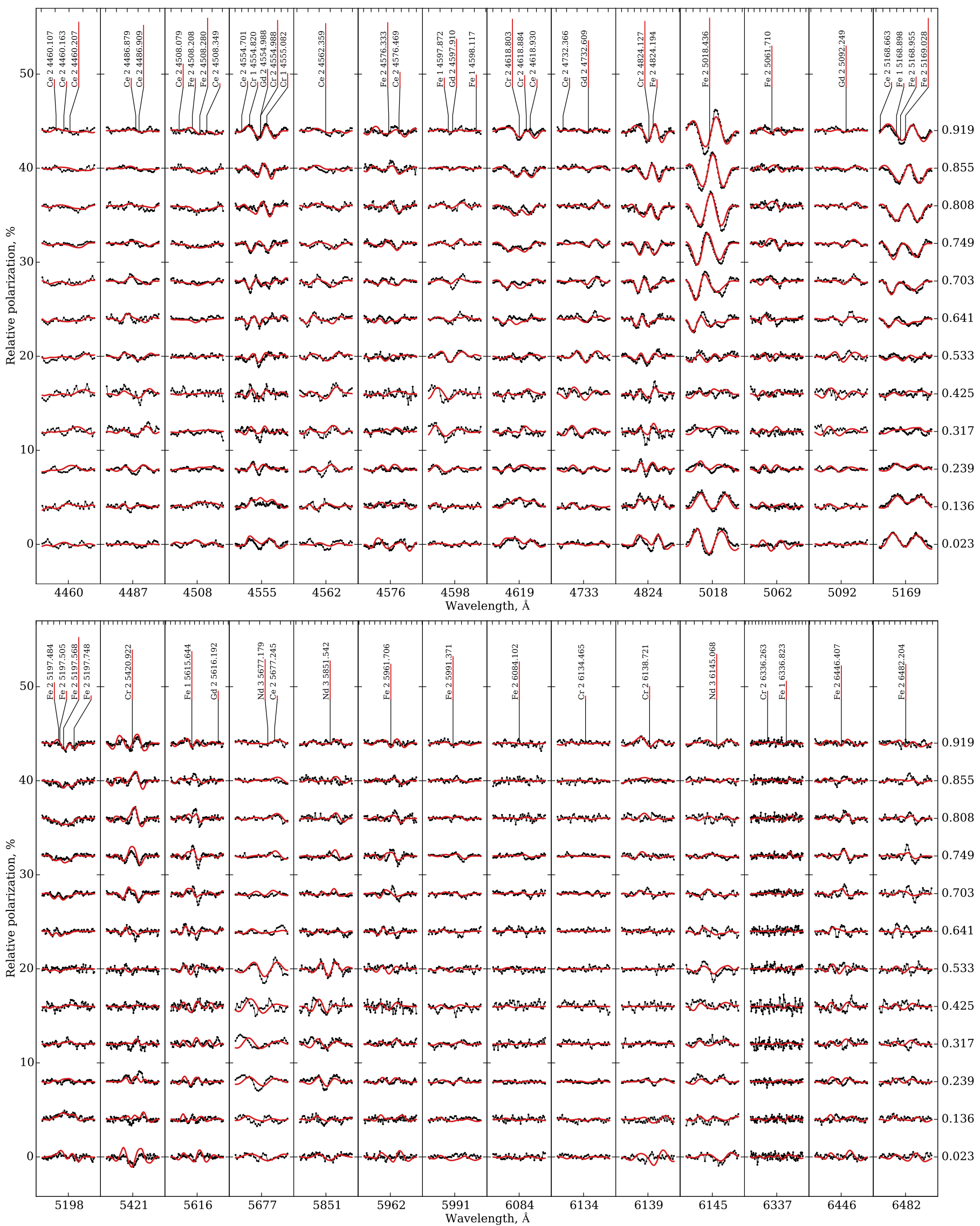

Fig. 10. Same as Fig. 9 for the Stokes $Q$ profiles. 


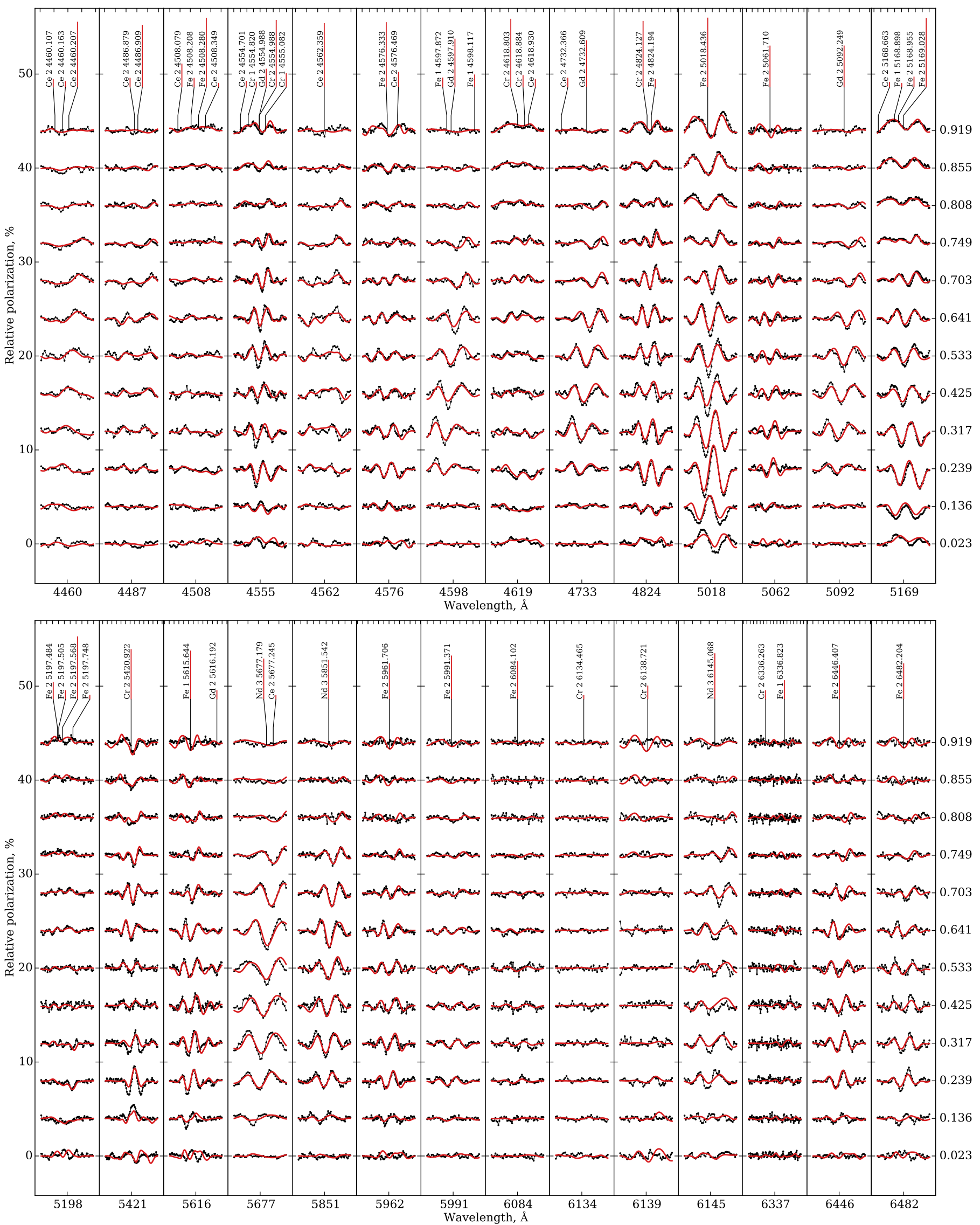

Fig. 11. Same as Fig. 9 for the Stokes $U$ profiles. 
:
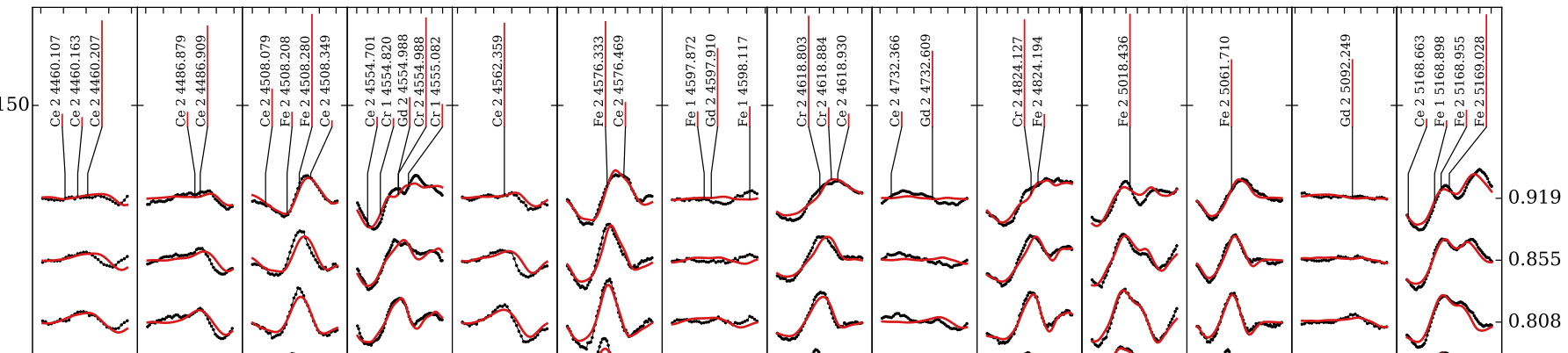
$\therefore$ -

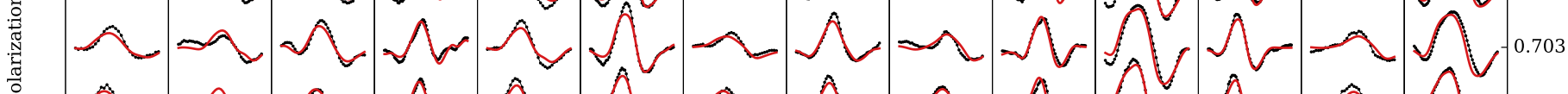

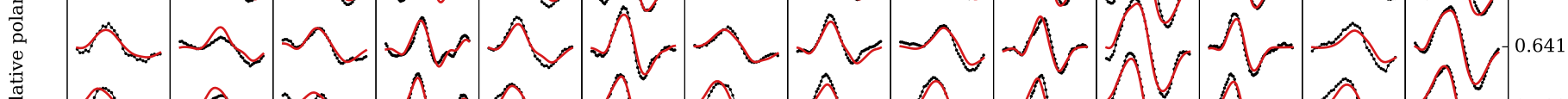

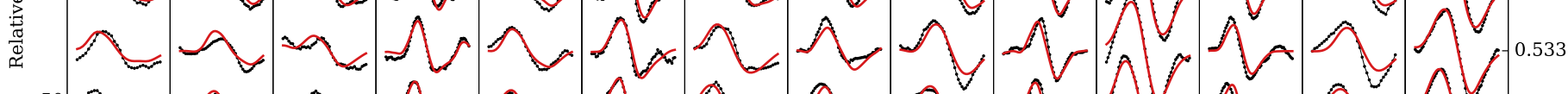

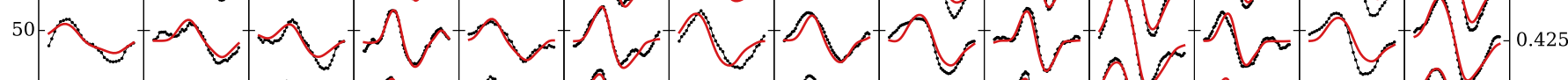

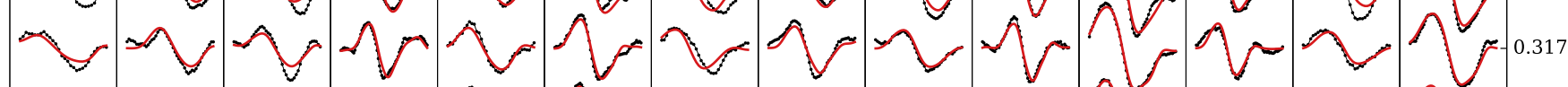

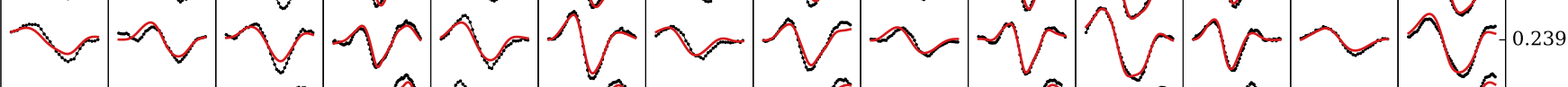

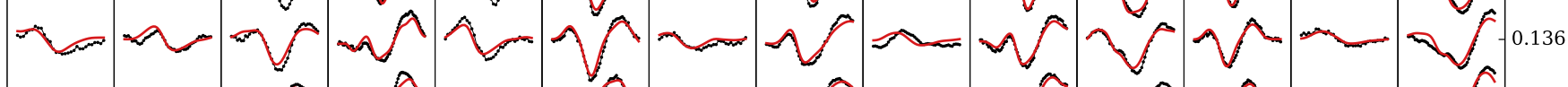

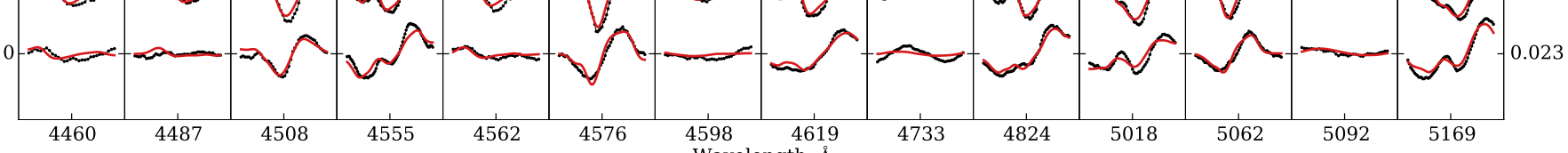

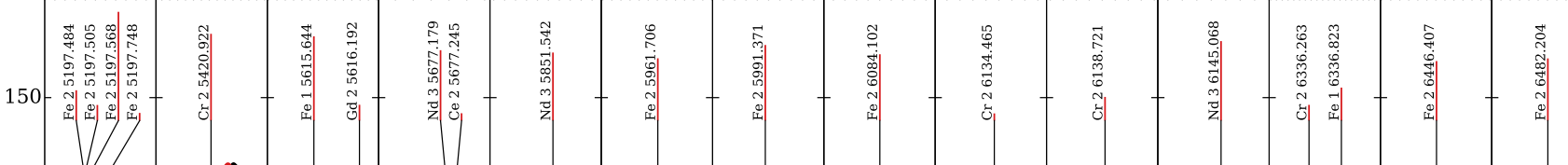

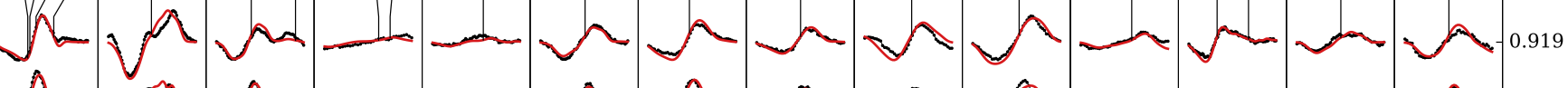

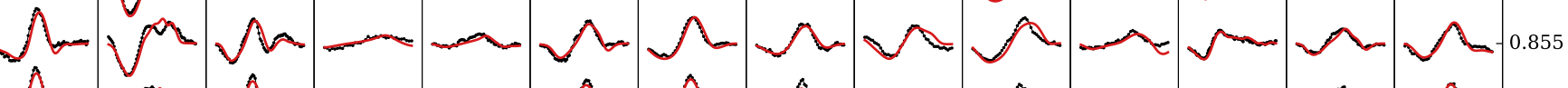

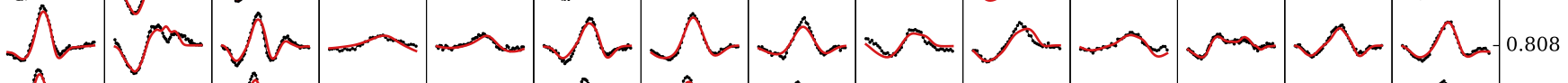

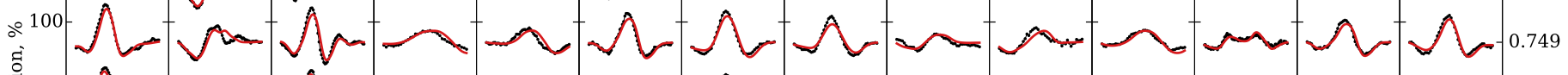
暮

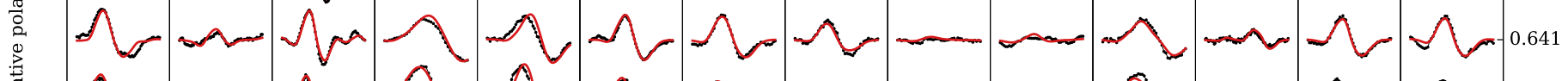

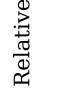
50

(n)

Fig. 12. Same as Fig. 9 for the Stokes $V$ profiles. 


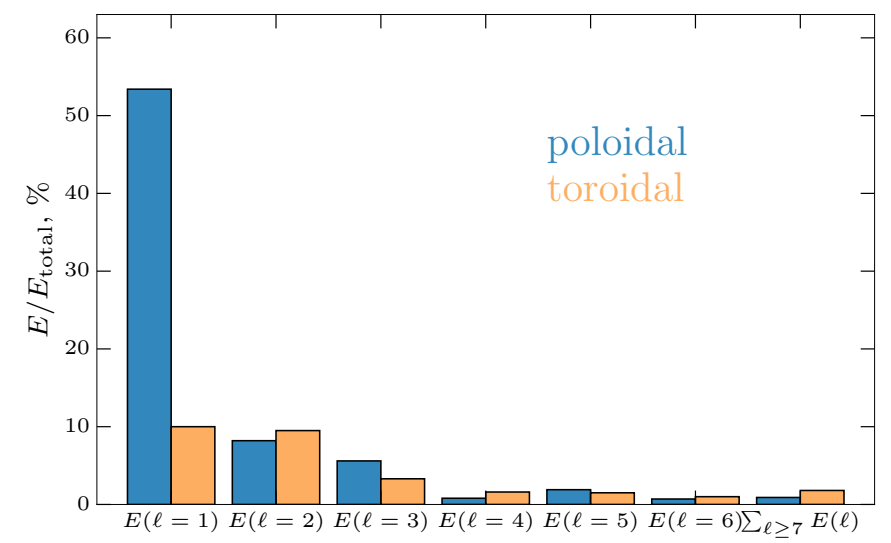

Fig. 13. Relative energies of the poloidal and toroidal harmonic modes for the magnetic field topology of HD 125248. The energy of the poloidal and toroidal components are shown in dark (blue) and light (orange) respectively. The last two bars represent the sum of energies for $\ell \geq 7$ modes.

The abundance distribution of Ti was derived by keeping the previously inferred magnetic field geometry and abundance maps of the other chemical elements fixed. The chemical abundance maps are presented in Fig. 15. The minimum and maximum abundance values were determined by taking the 5 and 95 percentiles for each map so that extreme outliers are excluded.

The resulting abundance maps show that HD125248 has very strong surface abundance inhomogeneities. The inferred map of Fe has abundance ranges from -2.9 to -4.1 in $\log \left(N_{\mathrm{Fe}} / N_{\mathrm{tot}}\right)$ units. The abundance map shows that Fe exhibits an underabundance arc that loosely correlates with the magnetic equator. We do not see any other correlations between the magnetic field topology and the abundance map of Fe.

The chromium abundance changes most dramatically with ranges from -2.7 to $-6.2 \mathrm{in} \log \left(N_{\mathrm{Cr}} / N_{\text {tot }}\right)$ units. The map of this element shows one very strong overabundance patch, which coincides with the magnetic field region of negative polarity. Such a dramatic 3.5 dex difference between the maximum and minimum value of $\mathrm{Cr}$ abundance is required to reproduce the behavior of the Stokes $I$ profiles of lines such as Cr II $\lambda 4555 \AA$ or Cr II $\lambda 6134 \AA$ for phases $\varphi \approx 0.5$. Thus, it is highly unlikely that the wide range of the abundance map of $\mathrm{Cr}$ is an artifact of our MDI procedure, and instead it reflects what is observed in the input spectra.

The surface abundance distributions of the rare earth elements for which we performed MDI do not follow the abundance patterns of $\mathrm{Cr}$ or Fe. Instead, rare earth elements are concentrated where the magnetic field is of positive polarity. The abundance maps of $\mathrm{Nd}$ and $\mathrm{Gd}$ describe well the strong changes of their Stokes $I$ profiles around phases $\varphi \approx 0$ when the line profiles of these elements become unusually shallow. The same can be said for line profiles of Ce ions, however, this element does not become strongly underabundant for phases close to zero as is the case for $\mathrm{Nd}$ and $\mathrm{Gd}$. For these three elements, the maximum abundance appears to be close to -6.35 , while their minimal value is between -8.1 and -8.4 in $\log \left(N_{\mathrm{X}} / N_{\text {tot }}\right)$ units.

The abundance distribution of $\mathrm{Ti}$ was derived from eight Ti II lines with strong polarization signatures and noticeable rotational modulation. The selection of these lines was performed on the basis of the same principles as the main line list (Sect. 5.2). However, in this case we also included $\mathrm{Ti}$ lines that were slightly blended by lines of $\mathrm{Fe}, \mathrm{Cr}$, and $\mathrm{Ce}$. The line selection for the mapping of $\mathrm{Ti}$ is presented in Table 7 . We derived the abundance
Table 7. Atomic data used in the abundance mapping of titanium.

\begin{tabular}{ccccc}
\hline \hline Ion & $\lambda(\AA)$ & $E_{i}(\mathrm{eV})$ & $\log g f$ & $\bar{g}$ \\
\hline Ti II & 4163.644 & 2.590 & -0.13 & 1.07 \\
Ti II & 4394.059 & 1.221 & -1.77 & 1.34 \\
Ti II & 4443.801 & 1.080 & -0.71 & 0.92 \\
Ti II & 4468.493 & 1.131 & -0.63 & 1.05 \\
Ti II & 4563.757 & 1.221 & -0.69 & 0.99 \\
Ti II & 4571.971 & 1.572 & -0.31 & 0.94 \\
Ti II & 4805.085 & 2.061 & -0.96 & 1.14 \\
Ti II & 5188.687 & 1.582 & -1.05 & 1.20 \\
\hline
\end{tabular}

Notes. Same as Table 6.

map of Ti with the magnetic field and abundance maps derived in the simultaneous $\mathrm{MDI}$ of $\mathrm{Fe}, \mathrm{Ce}, \mathrm{Nd}, \mathrm{Ce}$, and $\mathrm{Gd}$ as fixed parameters and allowing the INVERS 10 code to fit the selected Ti line profiles by finding a best-fit abundance map of this element. The resulting abundance map of $\mathrm{Ti}$ is illustrated in Fig. 15 (row six). From this figure we can see that Ti does not have strong abundance contrasts; its abundance ranges from -5.9 to -6.9 in $\log \left(N_{\mathrm{Ti}} / N_{\text {tot }}\right)$ units. Interestingly, the surface abundance pattern of Ti follows the rare earth elements instead of $\mathrm{Fe}$ or $\mathrm{Cr}$; a similar correlation between $\mathrm{Ti}$ and $\mathrm{Nd}$ has also been observed for $\alpha^{2}$ CVn (Silvester et al. 2014b).

The computed and observed line profiles of Ti are shown in Fig. 16. We can see that most polarization signatures present in the observations are reproduced by our code including the blending by spectral lines of other chemical elements. The Stokes $Q$ profiles of several $\mathrm{Ti}$ lines for phase $\varphi \approx 0.136$ are the only examples of a systematic discrepancy that we could find. This happens close to the phase when we see a sign change of the Stokes $U$ profiles for several lines, for example, Fe II $\lambda 5018 \AA$ and Fe II $\lambda 5169 \AA$. Similar behavior around this phase can also be noticed for the Ti Stokes $Q U$ profiles. We do not consider these minor discrepancies capable of invalidating our results for the following reasons. There are no strong systematic discrepancies between the observed and synthetic line profiles from which we determined the magnetic field structure. The synthetic Stokes $Q$ profiles for Ti have weaker amplitudes at phase 0.136 , while their morphology is correctly reproduced for other phases.

\section{Summary and discussion}

We have obtained spectra in all four Stokes parameters of the magnetic Ap star HD 125248. The spectropolarimetric observations were obtained with the HARPSpol instrument at the $3.6 \mathrm{~m}$ ESO telescope, and have a resolving power exceeding $10^{5}$ and $\mathrm{S} / \mathrm{N}$ of 200-300. The full set of spectropolarimetric observations is comprised of 36 individual Stokes parameter spectra and covers the entire rotational period of the star.

Based on these HARPSpol spectra, we calculated LSD profiles using different line masks. These profiles were used to obtain new precise measurements of the mean longitudinal magnetic field and net linear polarization. The longitudinal field measurements were combined with literature data to find an improved rotational period.

The atmospheric parameters of HD 125248 were derived with the help of modern atmosphere models, which include detailed treatment of nonsolar abundances and the presence of a strong magnetic field. Our abundance analysis of a large number of chemical elements showed that HD 125248 is deficient in 


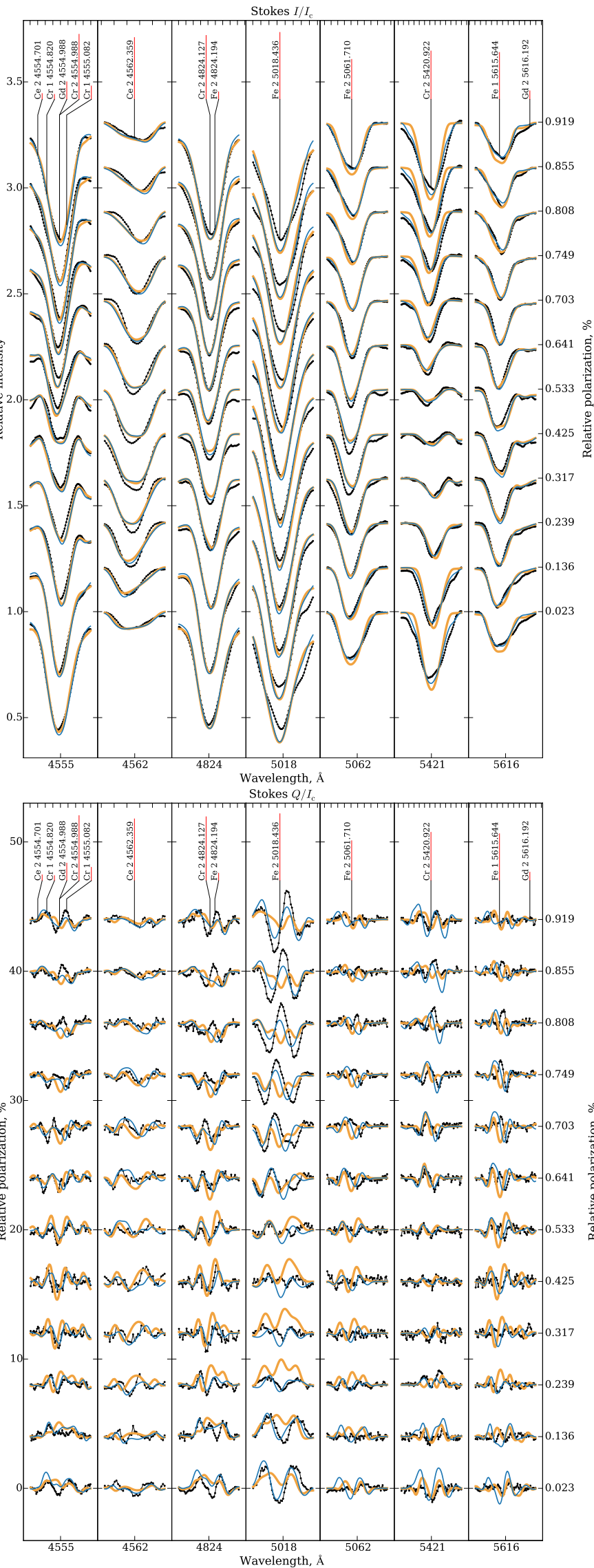

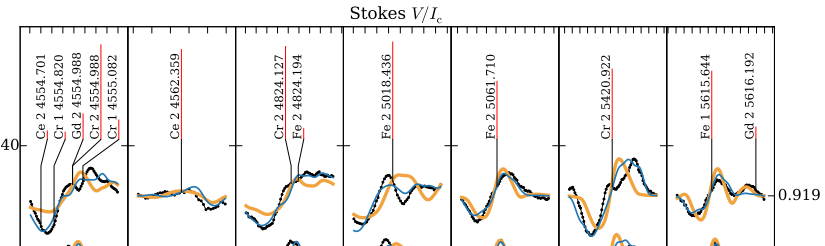

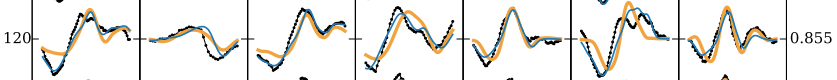

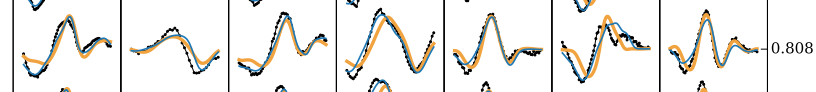

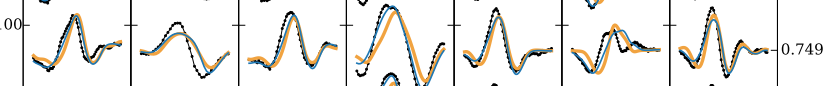

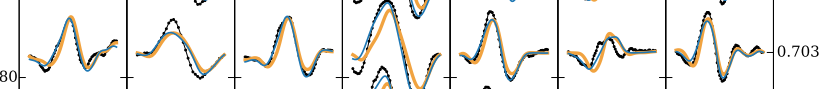

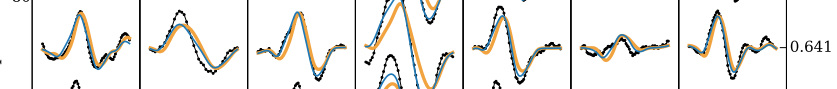

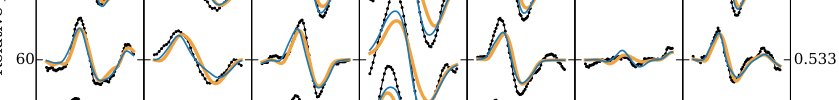

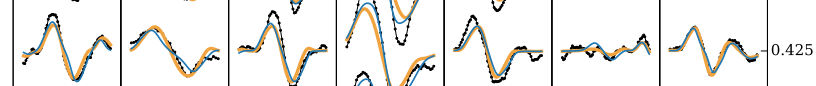
40

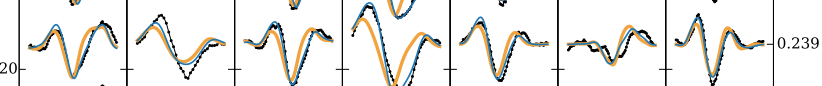
N

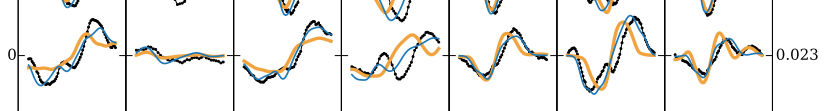

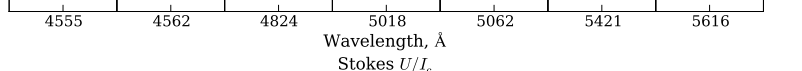

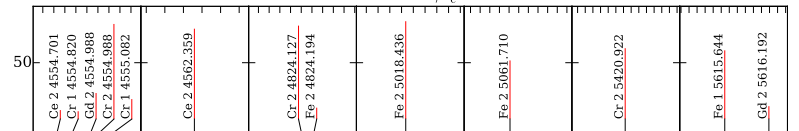

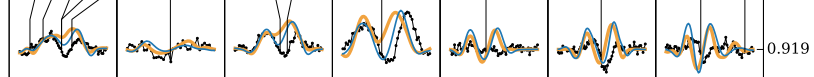

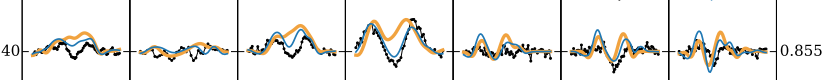

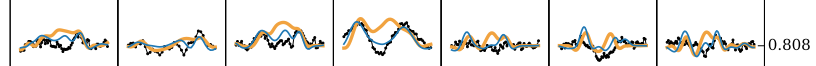

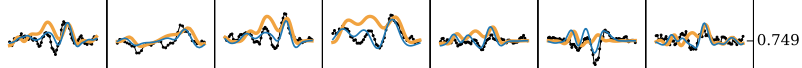

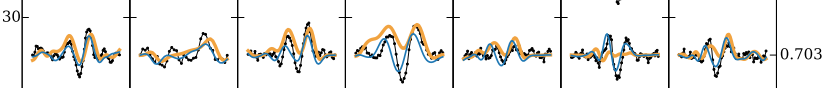

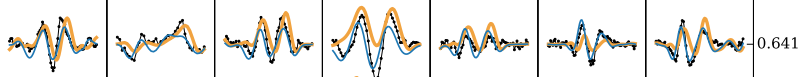
M Ma a a

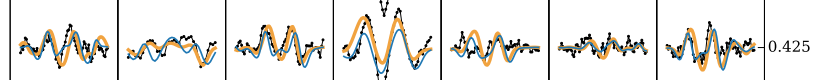

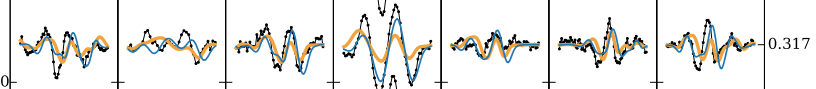

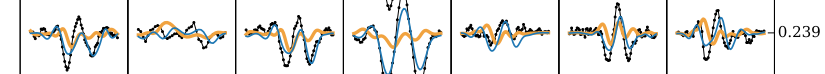

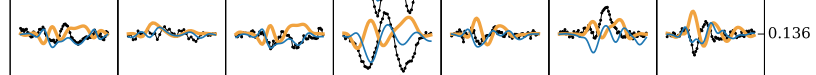

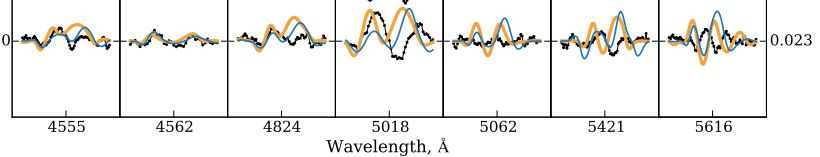

Fig. 14. Comparison of the observed (dots connected with black lines) and synthetic Stokes profiles calculated using dipole field (thick yellow lines) and a dipole plus quadrupole field (thin blue lines). The format of this figure is similar to Fig. 9. 
$\mathrm{Fe}$
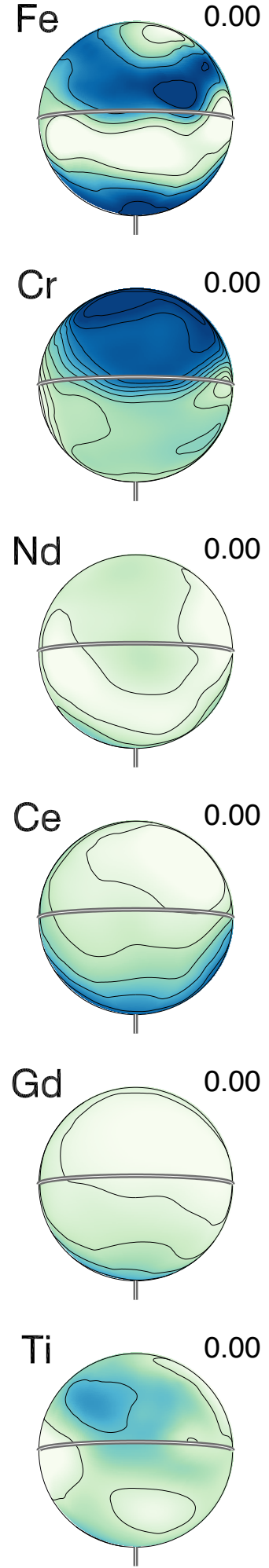

0.20
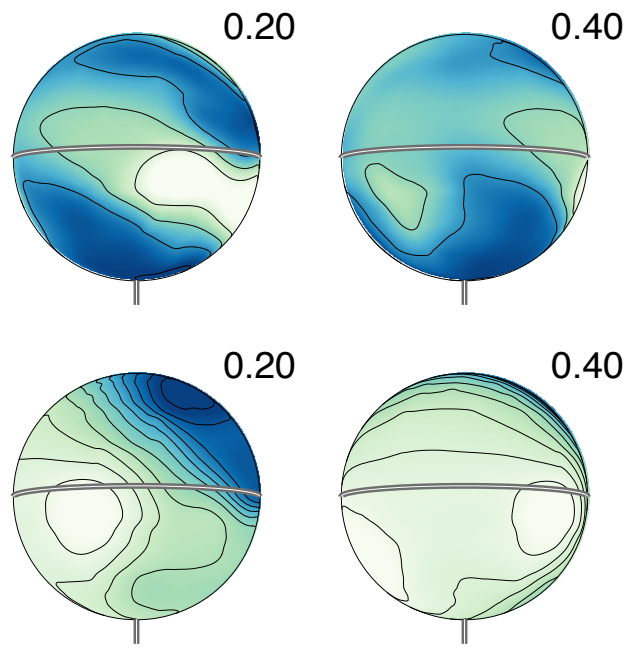

0.40

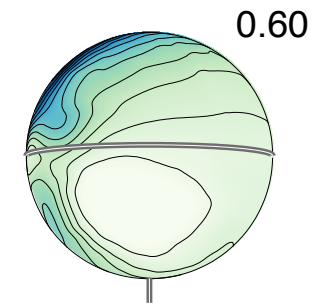

0.40
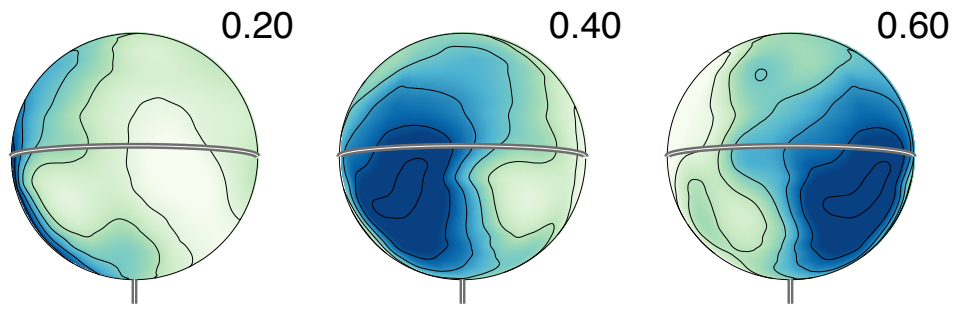

0.60

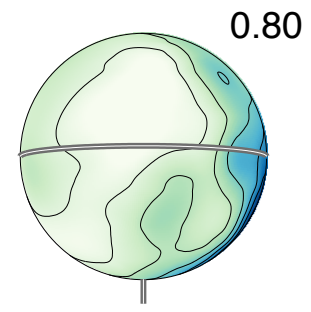

0.80

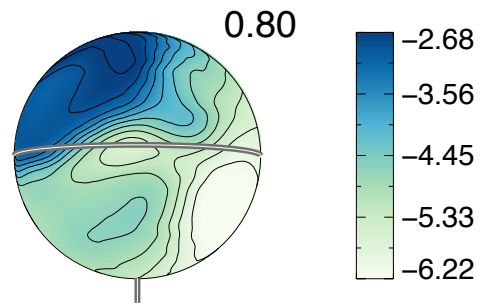

$-6.22$
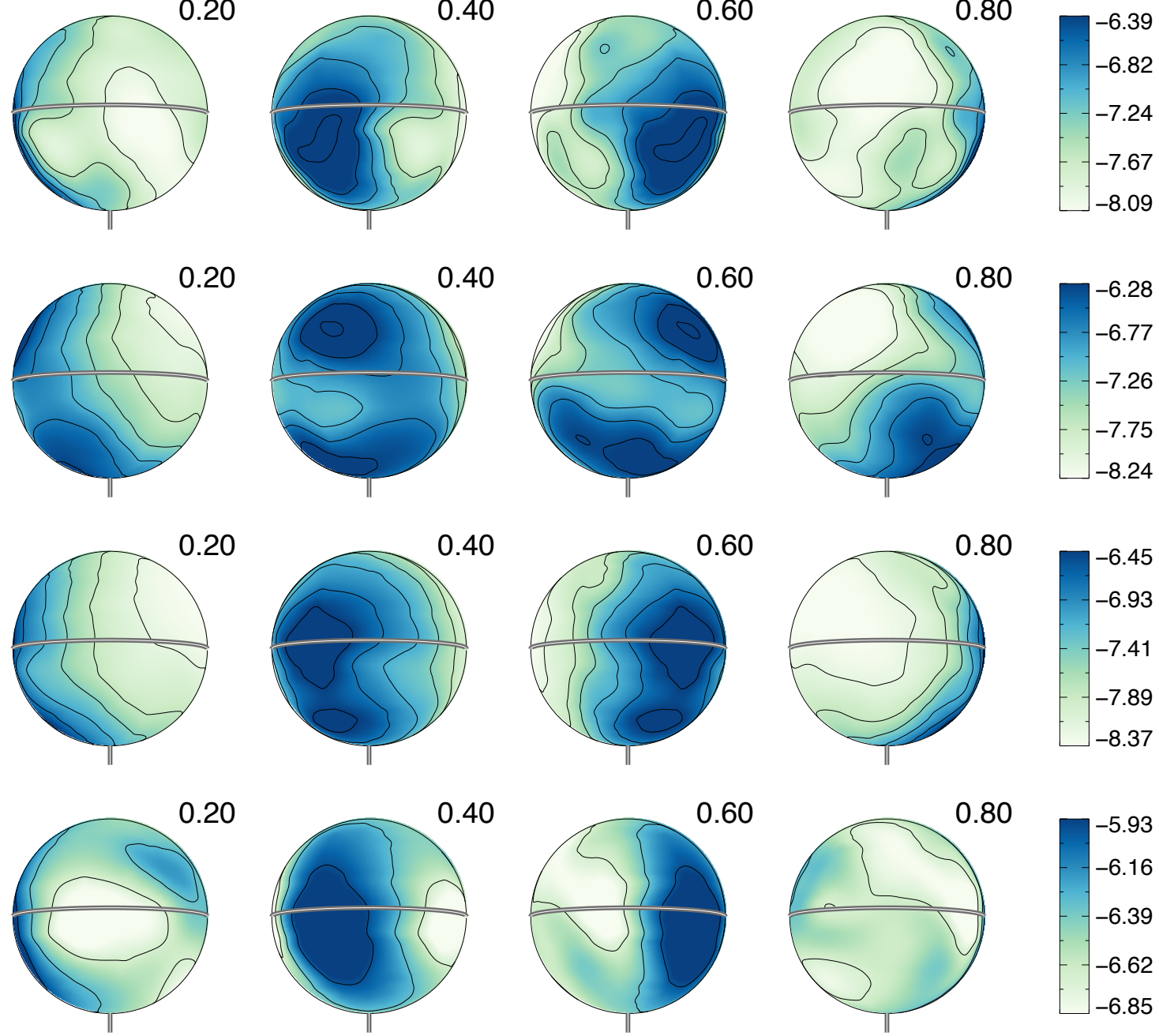

Fig. 15. Abundance distributions of $\mathrm{Fe}, \mathrm{Cr}, \mathrm{Nd}, \mathrm{Ce}, \mathrm{Gd}$, and $\mathrm{Ti}$ on the surface of $\mathrm{HD} 125248$. The color bars on the right indicate the abundance in $\log \left(N_{\mathrm{X}} / N_{\text {tot }}\right)$ units. The contours are plotted with 0.35 dex steps. The thick line and the vertical bar indicate positions of the rotational equator and the pole, respectively.

He and shows overabundances for most Fe-peak and rare earth elements. The abundance analysis of Fe II and Cr II lines indicates that HD 125248 has vertical abundance gradients in its atmosphere. We did not find strong difference in the abundances measured for different ions of the rare earth elements reported for cooler Ap stars by Ryabchikova et al. (2004).
The magnetic Doppler imaging of HD 125248 revealed a magnetic field that is dipole-like only on the largest spatial scales with strong field strength asymmetry between the two regions of opposite polarity and a major deviation from axisymmetry. Most of the magnetic field energy of HD 125248 is contained in the $\ell=1-3$ harmonic modes. Our analysis showed that 

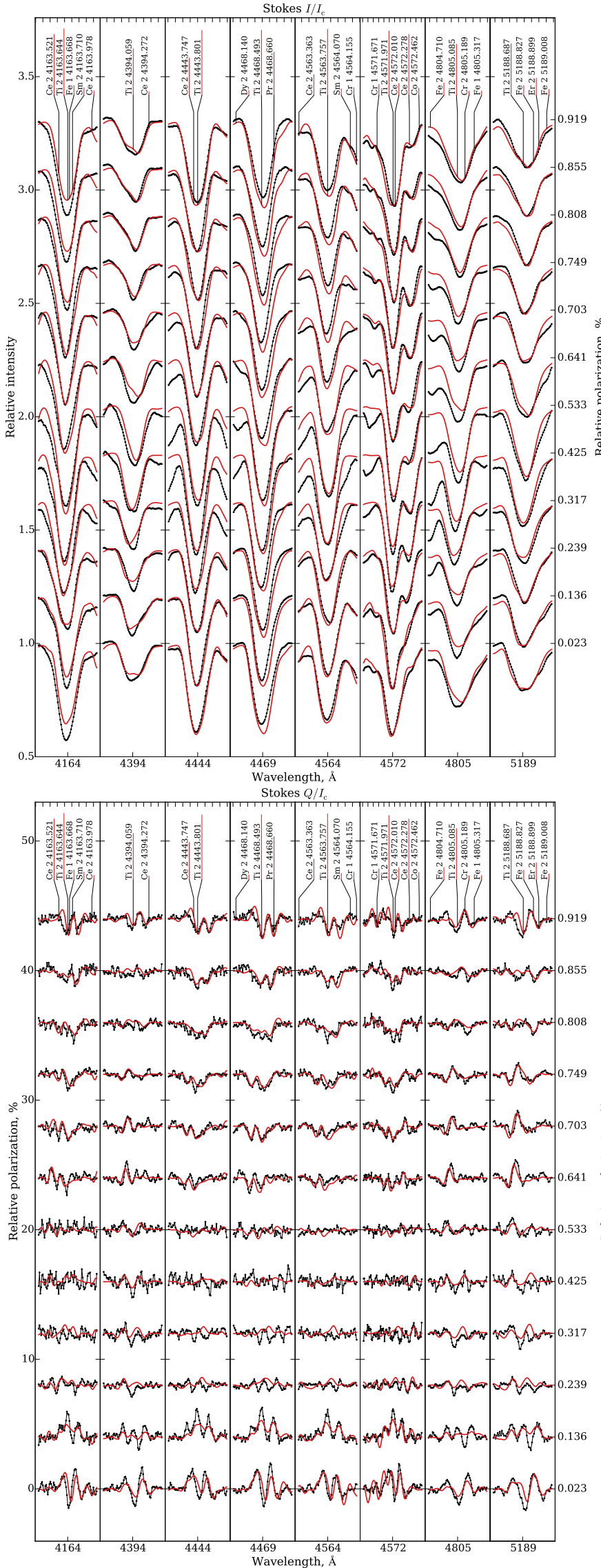

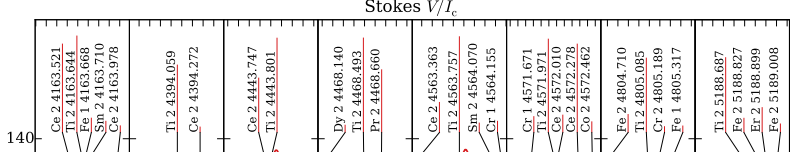

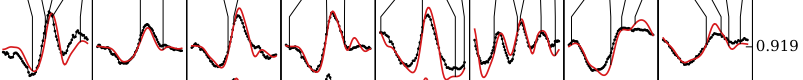

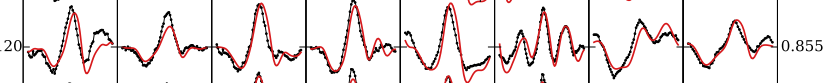

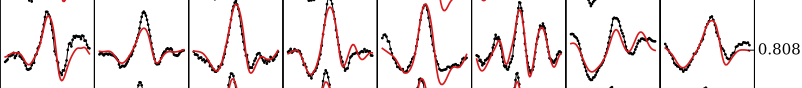

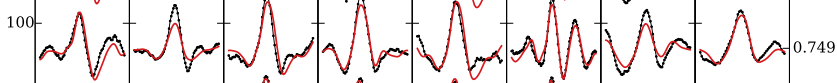

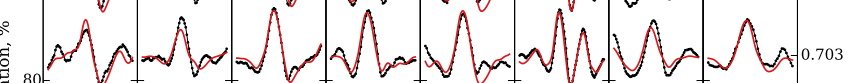

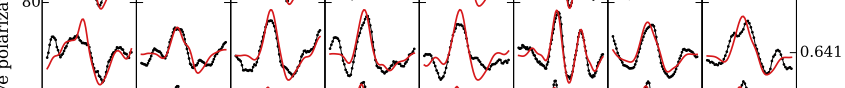

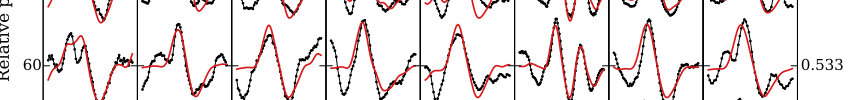

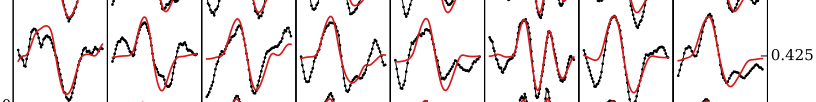
舟

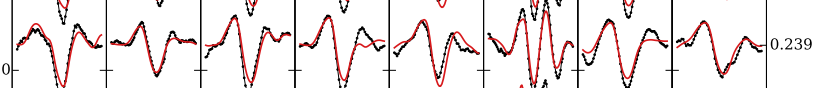

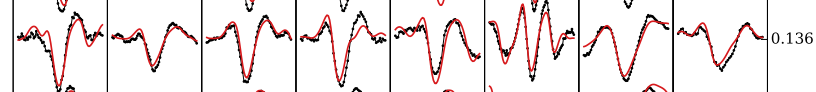

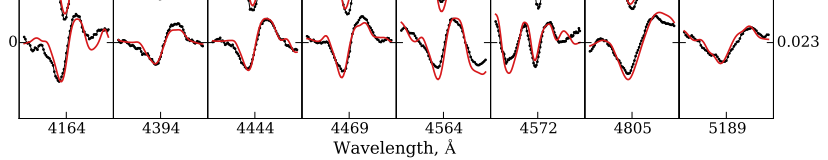

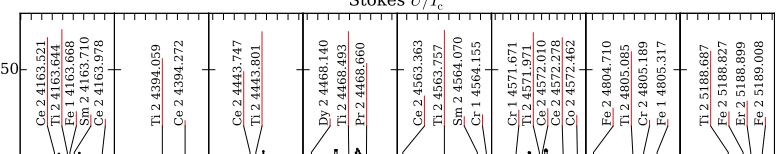

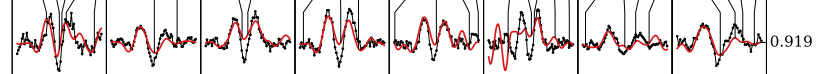
40 ond A

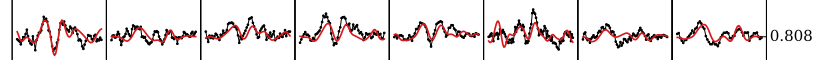

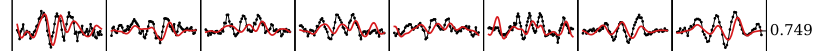

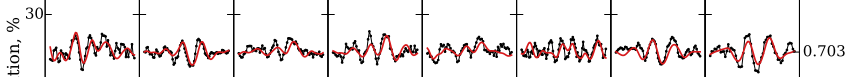

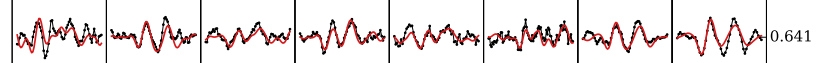

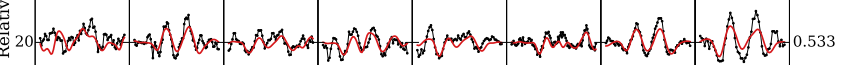

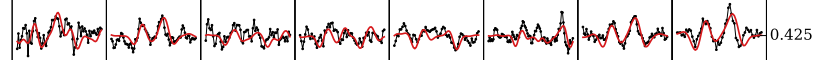

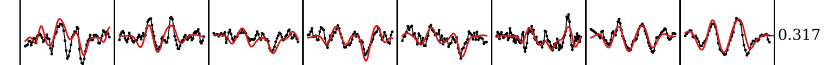
${ }_{4164}{ }_{4394}^{444}{ }_{\substack{4469 \\ \text { Wavelengt, } A}}^{4542}$

Fig. 16. Comparison of the observed (dots connected with black lines) and synthetic (thin red lines) Stokes profiles for Ti. The format of this figure is similar to Fig. 9. 
neither a purely dipole field nor a more complex dipole plus quadrupole field topology can reproduce the observations in all Stokes parameters.

The abundance distribution maps derived in the MDI analysis showed high contrast regions. However, we do not see an obvious correlation between horizontal field strength and overabundance patches as suggested by the recent theoretical studies of atomic diffusion in the presence of magnetic fields (Alecian \& Stift 2010; Alecian 2015). Instead, we see that $\mathrm{Cr}$ is most abundant where the field is of negative polarity, while the rare earth elements follow the field with positive polarity. The abundance of $\mathrm{Fe}$ does not correlate with the magnetic field in any obvious way except for a relative underabundance in the area of horizontal field.

Silvester et al. (2015) made a qualitative assessment of the complexity of the magnetic field of several Ap stars by comparing the distribution of the magnetic field energy derived from MDI studies as a function of angular degree to other stellar parameters. They found that a correlation between field complexity and stellar mass or age is probably present, as already suggested by Rusomarov et al. (2015).

Our results indicate that the magnetic field complexity of HD 125248 is similar to CU Vir (Kochukhov et al. 2014), $\alpha^{2}$ CVn (Silvester et al. 2014a), and HD 32633 (Silvester et al. 2015). These four stars are young objects with masses between 2.3 and $3.1 M_{\odot}$. Rusomarov et al. (2015) found a dipole field for HD 24712, which has a mass of $1.55 M_{\odot}$ and is likely to be much older than the other stars. Thus, it appears that our finding of magnetic field for HD 125248, which is significantly more complex than a dipole, supports the hypothesis that old (or less massive) Ap stars have predominantly dipolar fields with little structure on small scales. However, given the small sample of Ap stars for which full Stokes vector MDI studies have been carried out, it is difficult to make any definite conclusions about these trends. Nonetheless, as more four Stokes parameter MDI studies become available, we will be able to fully assess correlations between the field complexity and stellar parameters of magnetic Ap stars.

Acknowledgements. O.K. acknowledges financial support from the Knut and Alice Wallenber Foundation, the Swedish Research Council, and the Göran Gustafsson Foundation. The computations presented in this paper were performed on resources provided by SNIC through Uppsala Multidisciplinary Center for Advanced Computational Science (UPPMAX) under project p2013234. T.R. acknowledges partial financial support from the Presidium of RAS Program P-41. Resources provided by the electronic databases (VALD, Simbad, NASA ADS) are gratefully acknowledged. N.R. is profoundly grateful to J. Silvester for the deeply stimulating discussions.

\section{References}

Abt, H. A., \& Morrell, N. I. 1995, ApJS, 99, 135

Adelman, S. J., Pyper, D. M., Shore, S. N., White, R. E., \& Warren, Jr., W. H. 1989, A\&AS, 81, 221

Alecian, G. 2015, MNRAS, 454, 3143

Alecian, G., \& Stift, M. J. 2010, A\&A, 516, A53

Asplund, M., Grevesse, N., Sauval, A. J., \& Scott, P. 2009, ARA\&A, 47, 481

Babcock, H. W. 1947, PASP, 59, 260

Babcock, H. W. 1951, ApJ, 114, 1

Babcock, H. W. 1958, ApJS, 3, 141

Bagnulo, S., Landolfi, M., \& Landi Degl'Innocenti, M. 1999, A\&A, 343, 865

Bagnulo, S., Landi Degl' Innocenti, M., Landolfi, M., \& Mathys, G. 2002, A\&A, 394, 1023

Bagnulo, S., Landolfi, M., Landstreet, J. D., et al. 2009, PASP, 121, 993

Borra, E. F., \& Landstreet, J. D. 1980, ApJS, 42, 421
Braithwaite, J., \& Nordlund, Å. 2006, A\&A, 450, 1077

Catalano, F. A., Kroll, R., \& Leone, F. 1992, A\&A, 263, 203

Catalano, F. A., Leone, F., \& Kroll, R. 1998, A\&AS, 129, 463

Deutsch, A. J. 1947, ApJ, 105, 283

Deutsch, A. J. 1957, AJ, 62, 139

Donati, J.-F., \& Brown, S. F. 1997, A\&A, 326, 1135

Donati, J.-F., Semel, M., Carter, B. D., Rees, D. E., \& Cameron, A. C. 1997, MNRAS, 291, 658

Donati, J.-F., Howarth, I. D., Jardine, M. M., et al. 2006, MNRAS, 370, 629

Fossati, L., Ryabchikova, T., Bagnulo, S., et al. 2009, A\&A, 503, 945

Glagolevskij, Y. V. 2007, Astrophys. Bull., 62, 244

Gonzalez, J.-F., \& Artru, M.-C. 1994, A\&A, 289, 209

Hockey, M. S. 1969, MNRAS, 142, 543

Khan, S. A., \& Shulyak, D. V. 2006, A\&A, 454, 933

Kochukhov, O., \& Bagnulo, S. 2006, A\&A, 450, 763

Kochukhov, O., \& Piskunov, N. 2002, A\&A, 388, 868

Kochukhov, O., \& Wade, G. A. 2010, A\&A, 513, A13

Kochukhov, O., Bagnulo, S., Wade, G. A., et al. 2004, A\&A, 414, 613

Kochukhov, O., Makaganiuk, V., \& Piskunov, N. 2010, A\&A, 524, A5

Kochukhov, O., Lüftinger, T., Neiner, C., Alecian, E., \& MiMeS Collaboration 2014, A\&A, 565, A83

Kochukhov, O., Rusomarov, N., Valenti, J. A., et al. 2015, A\&A, 574, A79

Kupka, F., Piskunov, N., Ryabchikova, T. A., Stempels, H. C., \& Weiss, W. W. 1999, A\&AS, 138, 119

Landolfi, M., Landi Degl'Innocenti, E., Landi Degl'Innocenti, M., \& Leroy, J. L. 1993, A\&A, 272, 285

Landstreet, J. D., \& Mathys, G. 2000, A\&A, 359, 213

Landstreet, J. D., Borra, E. F., Angel, J. R. P., \& Illing, R. M. E. 1975, ApJ, 201, 624

Leone, F., \& Catanzaro, G. 2001, A\&A, 365, 118

Leroy, J. L. 1995, A\&AS, 114, 79

Lipski, Ł., \& Stępień, K. 2008, MNRAS, 385, 481

Lüftinger, T., Kochukhov, O., Ryabchikova, T., et al. 2010, A\&A, 509, A71

Mashonkina, L. I., Ryabtsev, A. N., \& Ryabchikova, T. A. 2002, Astron. Lett., 28,34

Mathys, G. 1992, A\&A, 256, L31

Mathys, G. 1994, A\&AS, 108, 547

Mathys, G. 1995, A\&A, 293, 746

Mathys, G., \& Hubrig, S. 1997, A\&AS, 124, 475

Mayor, M., Pepe, F., Queloz, D., et al. 2003, The Messenger, 114, 20

Michaud, G., Charland, Y., \& Megessier, C. 1981, A\&A, 103, 244

Mikulášek, Z., Janík, J., Zverko, J., et al. 2007, Astron. Nachr., 328, 10

Monier, R. 1992, A\&A, 263, 175

Morgan, W. W. 1931, ApJ, 74, 24

Perryman, M. A. C., Lindegren, L., Kovalevsky, J., et al. 1997, A\&A, 323, L49

Piskunov, N., \& Kochukhov, O. 2002, A\&A, 381, 736

Piskunov, N., Snik, F., Dolgopolov, A., et al. 2011, The Messenger, 143, 7

Renson, P., \& Manfroid, J. 2009, A\&A, 498, 961

Rusomarov, N., Kochukhov, O., Piskunov, N., et al. 2013, A\&A, 558, A8

Rusomarov, N., Kochukhov, O., Ryabchikova, T., \& Piskunov, N. 2015, A\&A, 573, A 123

Ryabchikova, T. 2014, in Putting A Stars into Context: Evolution, Environment, and Related Stars, Proc. of the Int. Conf., eds. G. Mathys, E. R. Griffin, O. Kochukhov, R. Monier, \& G. M. Wahlgren (Moscow: Publ. House Pero), 220

Ryabchikova, T., Nesvacil, N., Weiss, W. W., Kochukhov, O., \& Stütz, C. 2004, A\&A, 423, 705

Ryabchikova, T., Leone, F., \& Kochukhov, O. 2005, A\&A, 438, 973

Ryabchikova, T., Ryabtsev, A., Kochukhov, O., \& Bagnulo, S. 2006, A\&A, 456, 329

Ryabchikova, T., Piskunov, N., Kurucz, R. L., et al. 2015, Phys. Scr., 90, 054005

Shorlin, S. L. S., Wade, G. A., Donati, J.-F., et al. 2002, A\&A, 392, 637

Shulyak, D., Tsymbal, V., Ryabchikova, T., Stütz, C., \& Weiss, W. W. 2004, A\&A, 428, 993

Silvester, J., Kochukhov, O., \& Wade, G. A. 2014a, MNRAS, 440, 182

Silvester, J., Kochukhov, O., \& Wade, G. A. 2014b, MNRAS, 444, 1442

Silvester, J., Kochukhov, O., \& Wade, G. A. 2015, MNRAS, 453, 2163

Snik, F., Kochukhov, O., Piskunov, N., et al. 2011, in Solar Polarization 6, eds. J. R. Kuhn, D. M. Harrington, H. Lin, et al. ASP Conf. Ser., 437, 237

Stibbs, D. W. N. 1950, MNRAS, 110, 395

Tikhonov, A., \& Arsenin, V. 1977, Solutions of Ill-posed Problems, Scripta series in mathematics (Washington: Winston \& Wiley)

van Leeuwen, F. 2007, A\&A, 474, 653

Wade, G. A., Donati, J.-F., Landstreet, J. D., \& Shorlin, S. L. S. 2000, MNRAS, 313,823 